The Journal of Experimental Biology 212, 2691-2704

Published by The Company of Biologists 2009

doi:10.1242/jeb.022251

\title{
Biofluiddynamic scaling of flapping, spinning and translating fins and wings
}

\author{
David Lentink $^{1, *}$ and Michael H. Dickinson ${ }^{2}$ \\ ${ }^{1}$ Experimental Zoology Group, Wageningen University, 6709 PG Wageningen, The Netherlands and ${ }^{2}$ Department of Bioengineering, \\ California Institute of Technology, Pasadena, CA 91125, USA \\ *Author for correspondence (e-mail: david.lentink@wur.nl)
}

Accepted 5 February 2009

\begin{abstract}
SUMMARY
Organisms that swim or fly with fins or wings physically interact with the surrounding water and air. The interactions are governed by the morphology and kinematics of the locomotory system that form boundary conditions to the Navier-Stokes (NS) equations. These equations represent Newton's law of motion for the fluid surrounding the organism. Several dimensionless numbers, such as the Reynolds number and Strouhal number, measure the influence of morphology and kinematics on the fluid dynamics of swimming and flight. There exists, however, no coherent theoretical framework that shows how such dimensionless numbers of organisms are linked to the NS equation. Here we present an integrated approach to scale the biological fluid dynamics of a wing that flaps, spins or translates. Both the morphology and kinematics of the locomotory system are coupled to the NS equation through which we find dimensionless numbers that represent rotational accelerations in the flow due to wing kinematics and morphology. The three corresponding dimensionless numbers are (1) the angular acceleration number, (2) the centripetal acceleration number, and (3) the Rossby number, which measures Coriolis acceleration. These dimensionless numbers consist of length scale ratios, which facilitate their geometric interpretation. This approach gives fundamental insight into the physical mechanisms that explain the differences in performance among flapping, spinning and translating wings. Although we derived this new framework for the special case of a model fly wing, the method is general enough to make it applicable to other organisms that fly or swim using wings or fins.
\end{abstract}

Key words: fly, flight, swimming, wing, fin, scaling, biofluiddynamics, Navier-Stokes, dimensionless numbers.

\section{INTRODUCTION}

The use of dimensionless numbers for understanding complex biological flows has helped us enormously to better understand adaptations for swimming and flying in nature, as well as the corresponding flow phenomena. For example, the Reynolds number, $R e$, is the ratio of convective acceleration multiplied by density over viscous stress in the fluid (e.g. Tritton, 2005). It not only dictates what kind of propulsive mechanism the organism has at hand (viscous versus inertial) but also determines whether the flow is reversible or irreversible, or whether it is laminar or can become turbulent. Such dimensionless numbers can be interpreted in at least three ways: as ratios of force, time or length (e.g. Tennekes and Lumley, 1983). For example, although the Reynolds number is often described as the ratio of inertial to viscous forces, it can be interpreted as the ratio of convection length (or time) over diffusion length (or time) for a standard transport time (or distance) (e.g. Tennekes and Lumley, 1983). The wide-ranging use of the Reynolds number in biologically relevant flows is well illustrated in Steven Vogel's book 'Life In Moving Fluids' (Vogel, 1996).

The Strouhal number, St, is another important dimensionless number, which has been used extensively in the biological fluid dynamics literature. It is typically defined as $S t=f A / U$, where $f$ is flapping frequency, $A$ is flapping amplitude and $U$ is mean flow velocity. Its original context was as a measure of dimensionless shedding frequency for a bluff body undergoing von Kármán shedding in a constant flow (e.g. Guyon et al., 2001), but is has additional uses in biological fluid dynamics (Triantafyllou et al., 1993; Taylor et al., 2003). For example, it is proportional to the tangent of maximal induced angle of attack by a flapping wing or fin when the stroke plane is perpendicular to the direction of motion
(Taylor et al., 2003; Lentink et al., 2008). This amplitude-based Strouhal number closely resembles the inverse of the advance ratio $J=U / 2 \Phi f R$ as defined by Ellington (Ellington, 1984), where $\Phi$ is total wing beat amplitude in radians, $f$ is flapping frequency and $R$ is root-to-tip wing length. Note that $2 \Phi R$ is actually the total wingtip excursion in the stroke plane (downstroke plus upstroke), whereas $U / f$ measures wingbeat wavelength $\lambda$, the distance traveled during one stroke cycle. The advance ratio is therefore a measure of the pitch of a flapping wing; very much like the pitch of a propeller (and the pitch of a screw) provided that the stroke plane is normal to body speed. Dickinson (Dickinson, 1994) and Wang (Wang, $2000 \mathrm{~b}$ ) defined a chord-based Strouhal number $S t_{\mathrm{c}}=f c / U$, where $c$ is chord length. This number closely resembles the reduced frequency $k$ defined by Daniel and Webb (Daniel and Webb, 1987) for swimming $k=\pi f c / U$, which is usually defined as a ratio of velocity due to flapping to velocity due to forward motion. It should be noted that, in engineering fluid dynamics, Strouhal numbers are often reserved for their original purpose - to describe natural vortexshedding processes (e.g. Green, 1995) - whereas reduced frequencies (or the analogous dimensionless wavelength) are more appropriate for forced vibrations, such as flapping wings (e.g. Tobalske et al., 2007).

From our brief overview it becomes clear that different points of view exist in the biomechanics field on how to best define and use dimensionless numbers to study swimming and flight. Further, these dimensionless numbers are not always simple to interpret or, perhaps more importantly, similarly defined throughout the field. Our goal is to improve this for flapping studies by deriving a new set of dimensionless numbers. These numbers are not only directly linked to the Navier-Stokes (NS) equations but also can be 
interpreted more easily based on the morphology and kinematics of the wing (or fin). For this we chose to use morphological and kinematic length scale ratios, because they are most easy to interpret and illustrate geometrically.

For simplicity we focus our analysis on fly wings. In the discussion we will indicate how to apply the theory to the wings and fins of other organisms such as insects, birds, fish and samara seeds. First we derive the dimensionless NS equation with respect to the surface of the flapping wing of a forward flying fly. Next we show that the angle between the body velocity vector of a fly and its (approximate) stroke plane can be neglected for estimating the correct order of magnitude of the dimensionless numbers that depend on speed. We then further simplify the NS equation for hover conditions. Next we simplify the NS equations even further for spinning and translating fly wings. These more simplified forms of the NS equation and the corresponding dimensionless numbers are illustrated graphically for $3 \mathrm{D}$ wings. Our framework can therefore be readily applied to the design of appropriate parameter spaces for complex fluid dynamic studies of flapping, spinning and translating wings and fins. Finally we discuss how $3 \mathrm{D}$ and $2 \mathrm{D}$ wing kinematics are related and can potentially mediate the stall characteristics of a wing. Experimental tests of the efficacy of this new approach in characterizing salient features of biologically relevant forces and flows are presented in the accompanying paper (Lentink and Dickinson, 2009).

\section{MATERIALS AND METHODS \\ NS equation of a fly in forward flapping flight}

For problems related to flying and swimming, the relevant external media (air and water) are incompressible to within a good approximation. This significantly simplifies the governing equations such as those describing the conservation of mass (Eqn 1) and momentum (Eqn 2) (e.g. Anderson, 1991; White, 1991; Guyon et al., 2001; Tritton, 2005):

$$
\begin{gathered}
\boldsymbol{\nabla} \cdot \mathbf{u}=0, \\
\rho \frac{D \mathbf{u}}{D t}=-\nabla p+\mu \nabla^{2} \mathbf{u},
\end{gathered}
$$

in which

$$
\boldsymbol{\nabla}=\left[\begin{array}{l}
\partial / \partial x \\
\partial / \partial y \\
\partial / \partial z
\end{array}\right]
$$

is the gradient (del) operator, $\mathbf{u}$ is velocity, $\rho$ is density, $t$ is time, $p$ is pressure and $\mu$ is dynamic viscosity. Note that $\rho$ and $\mu$ are constant for incompressible air (and water). These equations are necessarily derived with respect to an inertial reference frame attached to earth. We can compute the time-dependent flow field, $\mathbf{u}(\mathbf{x}, t)$, around a flying fly using this system of time-dependent and non-linear partial differential equations ( $\mathbf{x}$ is the position vector in space). In order to solve the equations, we need to specify an initial velocity condition to start up the solution, and we need velocity boundary conditions to keep the solution within bounds (Fig. 1). An appropriate and simple initial condition is that the fly starts $(t=0)$ at rest in still air:

$$
\mathbf{u}(t=0)=\mathbf{0} .
$$

While we can further assume that the air far away from the fly remains practically unaffected by the fly's movement, on the outer boundary surface $S_{\mathrm{ob}}$ :

$$
\mathbf{u}\left(\mathbf{x} \in S_{\mathrm{ob}}\right)=\mathbf{0}
$$

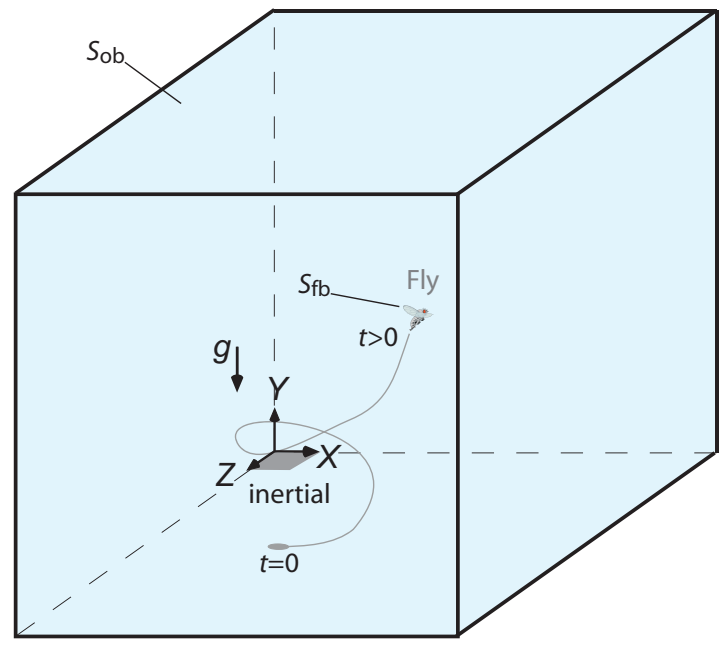

Fig. 1. Boundary and initial conditions of the Navier-Stokes (NS) equations of air around a fly. The fly flies with respect to an inertial coordinate system $(X, Y, Z)$ fixed to earth in a volume bounded by the outer surface $S_{o b}$ where the air remains at rest (outer boundary condition). The fly takes off at $t=0$ when the fly and fluid are at rest (initial condition). The air at the surface of the fly $S_{\mathrm{fb}}$ adopts the same velocity as the fly's body surface (inner boundary condition). Gravity is indicated with $g$.

Finally the air extremely close to the fly's outer surface $S_{\mathrm{fb}}$ can neither flow through nor slip with respect to the fly's surface; the air therefore adopts the same velocity as the fly's surface (e.g. White, 1991):

$$
\mathbf{u}\left(\mathbf{x} \in S_{\mathrm{fb}}\right)=\mathbf{u}_{\mathrm{fly}} .
$$

Although the initial condition (Eqn 3) and the boundary condition far away from the fly (Eqn 4) are simple because they are zero (homogeneous), the boundary condition at the fly's surface (Eqn 5) is not. The mathematical expression of the velocity boundary condition on the fly's surface must describe the fly's velocity distribution over its (microscopic) surface architecture, while tracking its position in space as the fly flies around. This formidable problem can be solved numerically using computational fluid dynamics (CFD) techniques (e.g. Liu and Kawachi, 1998; Wang, 2000a; Sun and Tang, 2002), or experimentally through building physical models of flying insects in the lab (Maxworthy, 1979; Dickinson, 1994; Ellington et al., 1996; Dickinson et al., 1999).

\section{Coordinate transformation that simplifies the fly's velocity boundary condition}

A complementary approach is to first transform the governing equations and boundary conditions (Eqns 1-5) such that the velocity boundary condition on the surface of interest is simplified. Preferably, the velocity boundary condition becomes zero such that we do not need to track the surface explicitly but implicitly through the coordinate transformation (e.g. Anderson, 1991; White, 1991; Vanyo, 1993; Guyon et al., 2001; Greitzer et al., 2004; Tritton, 2005). Such an approach is standard for studying cars, airplanes and even weather patterns on earth (e.g. Anderson, 1991; Batchelor, 2000). Transformation of the NS equation by placing the reference frame on a moving object such as an airplane (Fig. 2) seems almost trivial through its common use, e.g. in wind tunnels. Transformations of coordinate systems are similarly helpful when studying the flow around a propeller (Fig. 2) or turbine blade (e.g. Du and Selig, 1998; Dumitrescu and Cardos, 


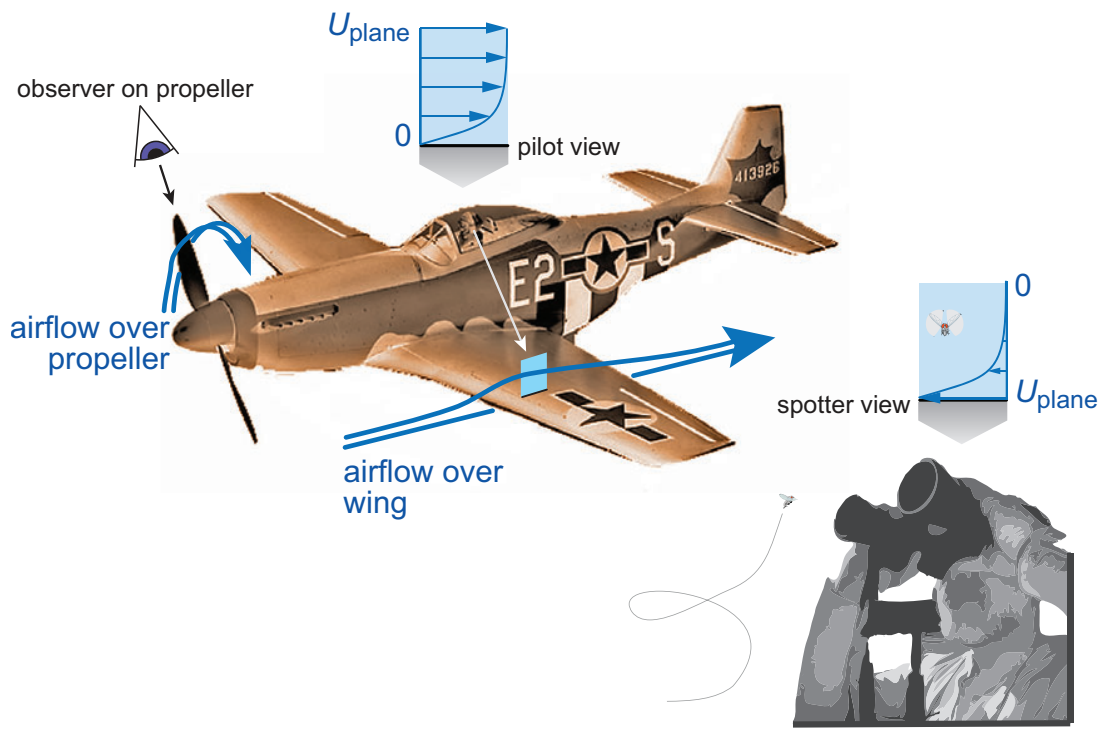

Fig. 2. The velocity profile in the boundary layer around an airplane wing depends on the observer; airplane spotter (inertial observer) versus pilot (local observer). In engineering problems it is customary to attach the reference frame (analogous to the observer) to the wing (like the pilot is). This transformation simplifies mathematical analysis, numerical simulations and experiments, e.g. through the use of wind tunnels. The main advantage is that the surface of interest remains stationary with respect to the reference frame. A similar approach can be used for propellers; in this case the reference frame (or unfortunate observer) is fixed to the spinning and forward moving propeller, which again simplifies analysis. Here we propose to use a similar approach for studying the aerodynamics of the even more complicated motion of flapping fly wings.
2003). Such an approach simplifies the mathematical analysis of the boundary layer flow, which mediates the shear stress and pressure distribution on the surface, and therefore the net aerodynamic force and moment.

Here we attach our local frame of reference $(x, y, z)$ to a flapping wing, at the wing's joint (Fig. 3). To simplify our analysis we assume that the fly flies along a straight path at constant speed in an arbitrary direction. In this transformation we neglect possible morphological undulations such as traveling waves in the wing, which holds because the amplitudes of undulations in a fly wing are typically small compared with the wing stroke amplitude. The kinematics of the wing, to which we attached our reference frame, consists of three rotational components; stroke $(\phi)$, deviation $(\varphi)$ and angle of attack $(\alpha)$ generate velocity gradients along the wing (Fig. 3A). The velocity transformation (Baruh, 1999) needed to make the velocity boundary condition identical to zero is (Vanyo, 1993; Greitzer et al., 2004):

$$
\mathbf{u}_{\text {inert }}=\mathbf{u}_{\text {loc }}+\left[\mathbf{u}_{\text {body }}+\boldsymbol{\Omega}_{\text {wing }} \times \mathbf{r}\right],
$$

in which $\mathbf{u}_{\text {inert }}, \mathbf{u}_{\text {loc }}$ and $\mathbf{u}_{\text {body }}$ are velocity with respect to the inertial (inert) and local (loc) coordinate system and the velocity of the fly's body, while $\boldsymbol{\Omega}_{\text {wing }}$ is the angular velocity of the fly wing and $\mathbf{r}$ is the position vector of a fluid particle in the rotating frame. The resulting transformed boundary conditions are:

$$
\begin{gathered}
\mathbf{u}_{\mathrm{loc}}(t=0)=\mathbf{0}, \\
\mathbf{u}_{\mathrm{loc}}\left(\mathbf{x} \in S_{\mathrm{ob}}\right)=-\left[\mathbf{u}_{\mathrm{body}}+\boldsymbol{\Omega}_{\mathrm{wing}} \times \mathbf{r}\right], \\
\mathbf{u}_{\mathrm{loc}}\left(\mathbf{x} \in S_{\mathrm{fb}}\right)=\mathbf{0} .
\end{gathered}
$$

The fluid acceleration with respect to the inertial frame $(X, Y, Z)$ $\mathbf{a}_{\text {inert }}$ is related to that in the rotating frame $\mathbf{a}_{\text {loc }}$ by the following transformation (Baruh, 1999; Vanyo, 1993; Greitzer et al., 2004):

$$
\mathbf{a}_{\text {inert }}=\mathbf{a}_{\mathrm{loc}}+\left[\mathbf{a}_{\mathrm{ang}}+\mathbf{a}_{\mathrm{cen}}+\mathbf{a}_{\mathrm{Cor}}\right]
$$

where

$$
\begin{gathered}
\mathbf{a}_{\text {ang }}=\dot{\boldsymbol{\Omega}} \times \mathbf{r}, \\
\mathbf{a}_{\mathrm{cen}}=\boldsymbol{\Omega} \times(\boldsymbol{\Omega} \times \mathbf{r}), \\
\mathbf{a}_{\mathrm{Cor}}=2 \boldsymbol{\Omega} \times \mathbf{u}_{\mathrm{loc}} .
\end{gathered}
$$

Here $\boldsymbol{\Omega}$ is the angular velocity and $\dot{\boldsymbol{\Omega}}$ is the angular acceleration of the rotating frame, and $\mathbf{r}$ and $\mathbf{u}_{\mathrm{loc}}$ are the position and velocity of a fluid volume in the rotating frame, respectively. The three terms enclosed in brackets in Eqn 10 are the angular $\left(\mathbf{a}_{\text {ang }}\right)$, centripetal $\left(\mathbf{a}_{\text {cen }}\right)$ and Coriolis $\left(\mathbf{a}_{\text {Cor }}\right)$ accelerations (note that we neglected the accelerations of the body itself because we assume steady flight. We now substitute the coordinate transformation described by Eqn 10 into the NS equation, Eqn 2, and obtain its form with respect to the local coordinate system $(x, y, z)$ fixed to the wing (Eqn 14). Note that $D \mathbf{u} / D t=\mathbf{a}_{\text {inert }}$ in Eqn 2, whereas $D \mathbf{u} / D t=\mathbf{a}_{\text {loc }}$ in Eqn 14, because we drop the 'inert' and 'loc' subscripts in the NS equation for easy notation in every subsequent NS equation:

$$
\rho \frac{D \mathbf{u}}{D t}+\rho \dot{\boldsymbol{\Omega}} \times \mathbf{r}+\rho \boldsymbol{\Omega} \times(\boldsymbol{\Omega} \times \mathbf{r})+\rho 2 \boldsymbol{\Omega} \times \mathbf{u}=-\nabla p+\mu \boldsymbol{\nabla}^{2} \mathbf{u} .
$$

This equation captures the (relative) accelerations and stresses that a 'fluid particle' with local velocity $\mathbf{u}$ experiences close to the wing (i.e. in its boundary layer). We consider accelerations (Eqns 10-13) instead of the analogous 'fictitious forces', which point in the opposite direction (e.g. Vanyo, 1993; Greitzer et al., 2004).

\section{Scaling the NS equation in the reference frame attached to the fly wing}

To find out how these acceleration and stress terms influence the physical phenomena around a fly wing, we scale all terms in Eqn 14 with respect to their orders of magnitude:

$$
\begin{aligned}
& \mathbf{u}^{*}=\frac{\mathbf{u}}{U}, t^{*}=\frac{U t}{c}, \nabla^{*}=c \cdot \nabla, \dot{\boldsymbol{\Omega}} *=\frac{\dot{\boldsymbol{\Omega}}}{\dot{\mathbf{\Omega}}}, \\
& \mathbf{\Omega}^{*}=\frac{\boldsymbol{\Omega}}{\Omega}, \mathbf{r}^{*}=\frac{\mathbf{r}}{R}, p^{*}=\frac{p}{p_{0}},
\end{aligned}
$$

in which * indicates a scaled, dimensionless, variable. The velocity u scales with the (absolute) time-averaged speed of the flapping wing at its wingtip $U$. The del operator $\nabla$ scales with the average chord length as $1 / c$, for which we divided the single-wing area $S$ by its single-wing span $b_{\mathrm{s}}\left(c=S / b_{\mathrm{s}}\right.$; Fig. 3B). Time $t$ scales with the time it takes the fluid to travel over the wing chord $c / U$ (e.g. Anderson, 1991; Tritton, 2005). The angular acceleration $\dot{\boldsymbol{\Omega}}$ and velocity $\boldsymbol{\Omega}$ scale with the (absolute) time-averaged angular acceleration amplitude $\dot{\Omega}$ and time-averaged angular velocity amplitude $\Omega$ of the wing, 


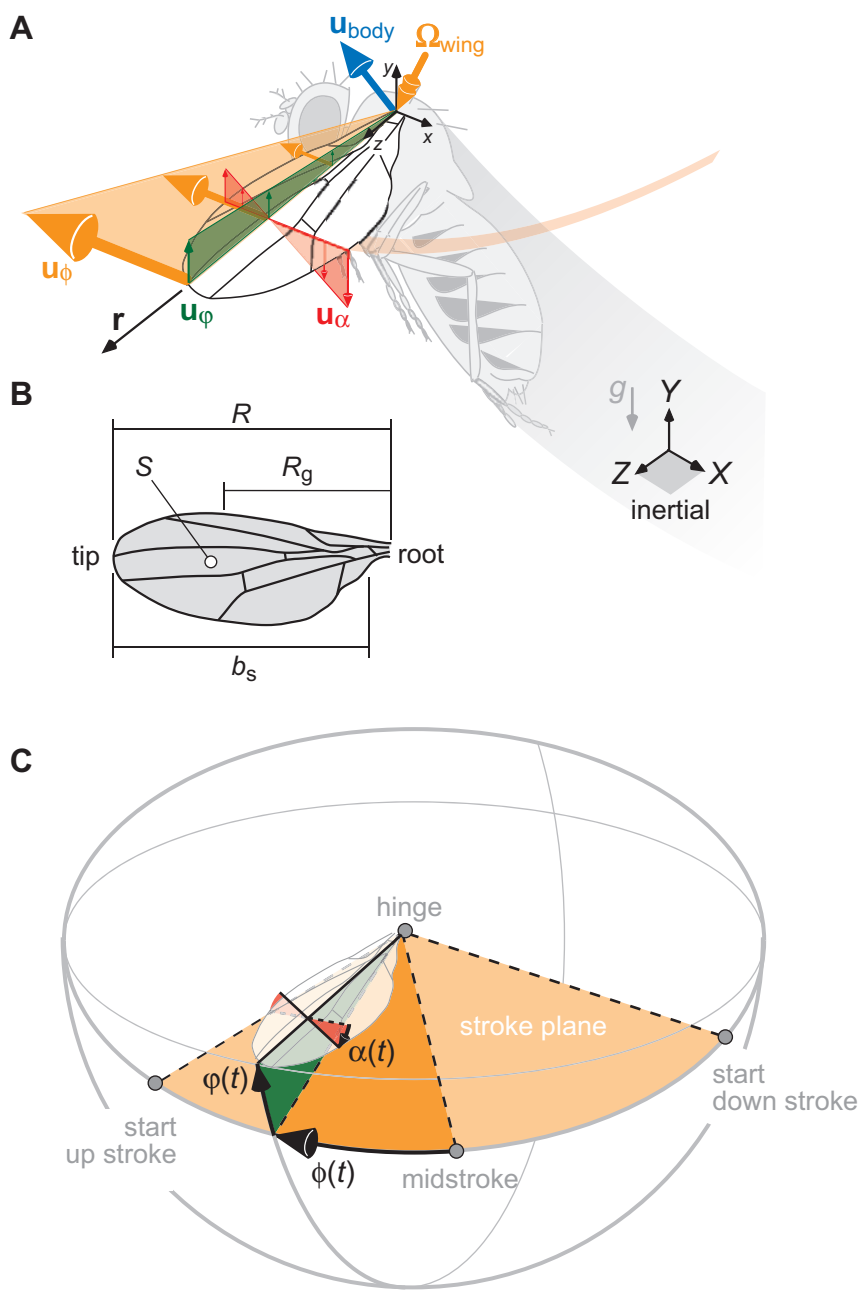

Fig. 3. The kinematics and morphology of a forward flying fly. (A) The fly flies with velocity $\mathbf{u}_{\text {body }}$ while flapping its wings with angular velocity $\boldsymbol{\Omega}_{\text {wing. }}$. The cross-product of the wing angular velocity vector and the local radius $\mathbf{r}$ induces three velocity gradients along the wing; a velocity gradient due to deviation $\mathbf{u}_{\varphi}$, stroke $\mathbf{u}_{\phi}$ and angle of attack variation $\mathbf{u}_{\alpha}$. There are two reference frames, the inertial reference frame $(X, Y, Z)$ attached to earth and the local reference frame $(x, y, z)$ attached to the fly's wing at the joint. (B) Wing morphology. Wing radius is the radial distance between the wing root and tip, $R$. The wing radius of gyration $R_{\mathrm{g}}$ can be calculated using a blade element method and is roughly equal to half the wing radius (Ellington, 1984). The average chord length of the wing $c$ can be calculated by dividing the single-wing area $S$ by the single-wing span $b_{\mathrm{s}}$. We define the single-wing aspect ratio as $R / c$. (C) The definition of wing deviation $\varphi$, stroke $\phi$ and angle of attack $\alpha$, which depend on time $t$ (Sane and Dickinson, 2001).

respectively. The local radius $\mathbf{r}$ scales with the wingtip radius, $R$, and the pressure $p$ scales with the ambient (atmospheric) pressure $p_{0}$. After substituting Eqns 15-21 into Eqn 14 we normalize the resulting equation by dividing it by the order of magnitude of the convective acceleration term of the fluid $\rho U^{2} / c$ (e.g. Anderson, 1991; Tritton, 2005; Greitzer et al., 2004). The resulting equation is dimensionless; for simplicity we omit *:

$$
\begin{array}{r}
1 \cdot \frac{D \mathbf{u}}{D t}+\frac{\dot{\Omega} R c}{U^{2}} \cdot \dot{\boldsymbol{\Omega}} \times \mathbf{r}+\frac{\Omega^{2} R c}{U^{2}} \cdot \boldsymbol{\Omega} \times(\boldsymbol{\Omega} \times \mathbf{r})+\frac{\Omega^{2} R c}{U^{2}} \cdot 2 \boldsymbol{\Omega} \times \mathbf{u}= \\
-\frac{p_{0}}{\rho U^{2}} \cdot \nabla p+\frac{\mu}{\rho U c} \cdot \nabla^{2} \mathbf{u} .
\end{array}
$$

This equation estimates the acceleration and stress that the flow experiences near the wing, where $\boldsymbol{\Omega}$ and $\dot{\boldsymbol{\Omega}}$ are roughly perpendicular to $\mathbf{r}$. Extra care had to be taken in handling the Coriolis term, which results from a cross-product between $\boldsymbol{\Omega}$ and $\mathbf{u}$, because the direction of the velocity $\mathbf{u}$ above the wing's surface consists of two components with different directions with respect to $\Omega$ : $\mathbf{u} \propto\left[\mathbf{u}_{\text {body }}+\boldsymbol{\Omega}_{\text {wing }} \times \mathbf{r}\right]$ (similar to Eqn 8 ). The first velocity component is induced by the fly's body motion, while the second component is induced by the flapping motion of the fly's wing. By taking the relative direction of $\mathbf{u}$ and $\boldsymbol{\Omega}$ into account we find that the Coriolis term is dominated by the velocity component of flow induced by wing beat $\boldsymbol{\Omega}_{\text {wing }} \times \mathbf{r}$, not by body motion $\mathbf{u}_{\text {body }}$, which holds for animals in general. The reason is that if the body velocity had a large component parallel to the stroke plane, which is needed for a significant contribution to the cross-product, it could induce flow stagnation, or even flow reversal, over the wing during either the downstroke or the upstroke. Flow stagnation or reversal typically compromises both lift generation and power efficiency (see Appendix I). Hence Coriolis acceleration typically scales with $\Omega^{2} R$ instead of $\Omega U$ for animals (and helicopters). The scale factors in Eqn 22 give the relative order of magnitude of all accelerations and stresses acting on an infinitesimal volume of fluid, compared with the convective acceleration. Hence these dimensionless scale factors enable us to pin point how the corresponding terms mediate aerodynamic mechanisms that govern flight.

\section{RESULTS}

Basic kinematic model of a fly wing in forward flight

The dimensionless numbers that scale the aerodynamics of a fly wing can be derived without loss of generality if we simplify the flight conditions and kinematics. This works because the order of magnitude of the scale factors is more important than their precise value. Mathematically our insect flight model is simplified in three steps. (1) Flight without sideslip (Fig. 4A). (2) Straight flight at constant speed $U_{\infty}$ in an arbitrary direction with respect to gravity (Fig. 4B). (3) Zero wing deviation $\varphi$ (Eqn 23) and sinusoidal wing stroke $\phi$ (Eqn 24) and angle of attack $\alpha$ (Eqn 25) kinematics:

$$
\begin{gathered}
\varphi=0, \\
\phi=\Phi_{0} \sin (2 \pi f t), \\
\alpha=\alpha_{0} \cos (2 \pi f t),
\end{gathered}
$$

in which the wing stroke and angle of attack flap $90^{\circ}$ out of phase at the same frequency $f$. Note that $\Phi_{0}$ is stroke amplitude, which is half the full amplitude defined by Ellington (Ellington, 1984), and $\alpha_{0}$ is angle of attack amplitude (the ranges found for insects are $0^{\circ}<\Phi_{0}<90^{\circ}$ and $0^{\circ}<\alpha_{0}<90^{\circ}$ ). In our simplification we use Ellington's observation that the wing kinematics of many insects can be approximated well with sinusoidal stroke kinematics, and that wing deviation with respect to the stroke plane is typically small (Ellington, 1984). This has also been found by Fry and co-workers for fruit flies (Fry et al., 2003). Based on Eqns 23-25 we calculated the resulting angular velocities and accelerations as:

$$
\begin{gathered}
\dot{\phi}=\Phi_{0} 2 \pi f \cos (2 \pi f t), \\
\ddot{\phi}=-\Phi_{0}(2 \pi f)^{2} \sin (2 \pi f t), \\
\dot{\alpha}=-\alpha_{0} 2 \pi f \sin (2 \pi f t), \\
\ddot{\alpha}=-\alpha_{0}(2 \pi f)^{2} \cos (2 \pi f t),
\end{gathered}
$$


A
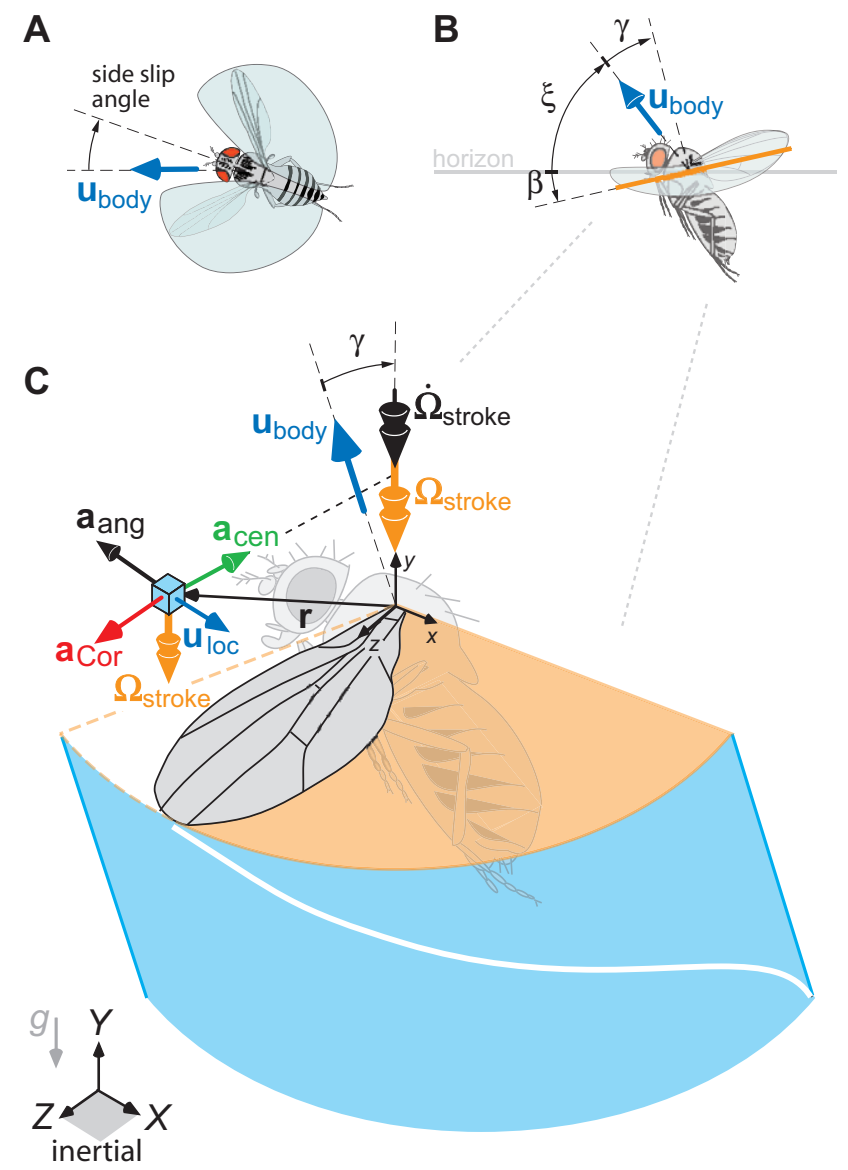

Fig. 4. Simplified forward flight model of a fly. (A) We first assume that the side slip angle of the fly, with respect to its body velocity, is zero.

(B) Definition of the stroke plane angle $\beta$ (Ellington, 1984), flight path angle $\xi$ (Ellington, 1984) and the angle between the normal vector of the stroke plane and the body velocity $\gamma$. (C) A hovering fly induces three rotational accelerations in the flow due to stroke (downstroke shown). Shown are the angular $\mathbf{a}_{\text {ang }}$, centripetal $\mathbf{a}_{\text {cen }}$ and Coriolis $\mathbf{a}_{\mathrm{cor}}$ accelerations that are induced in the fluid near the wing and result from the wing stroke, its propeller-like swing. The angular velocity of the wing stroke is $\Omega_{\text {stroke }}$ and its angular acceleration is $\dot{\Omega}_{\text {stroke. }}$. The local velocity of fluid with respect to the local reference frame $(x, y, z)$ is $\mathbf{u}_{\text {loc }}$. We color coded the stroke plane of the wing orange and the 'outer shell' blue, which features the white wingtip path. (Note that the depicted direction of angular acceleration was chosen to prevent image clutter.)

where $\dot{\phi}$ and $\ddot{\phi}$ are the first and second time derivative of wing stroke angle, respectively, and $\dot{\alpha}$ and $\ddot{\alpha}$ are the first and second time derivative of wing angle of attack, respectively.

\section{Visualizing rotational flow accelerations due to wing stroke}

For many insects, including flies, the velocity and acceleration due to wing stroke are larger than the velocity and acceleration due to angle of attack variation, because $\Phi_{0} R>\alpha_{0} c$ holds. Using Eqns 11-13 we can draw and interpret the rotational accelerations that result from the wing stroke and act on the fluid near the wing (Fig. 4C). The first component $\mathbf{a}_{\text {ang }}$ is the manifestation of the angular acceleration of the wing around its base, which results locally in a chord-wise acceleration (Fig. 4C). The second term $\mathbf{a}_{\text {cen }}$ represents the centripetal acceleration, which is directed spanwise towards the wing's base (Fig. 4C). The third term $\mathbf{a}_{\text {Cor }}$ represents the Coriolis acceleration; its direction depends on the direction of local fluid velocity $\mathbf{u}_{\text {loc }}$ (Fig. 4C). The precise directions of $\mathbf{a}_{\text {ang }}, \mathbf{a}_{\text {cen }}$ and $\mathbf{a}_{\text {Cor }}$ for insect kinematics also depend on contributions from wing deviation and rotation. We omitted these additional contributions in Fig. 4C, because they are generally smaller than the contribution from stroke and we wanted to keep the figure simple enough to interpret. Both the centripetal and Coriolis accelerations $\left(\mathbf{a}_{\mathrm{cen}}\right.$ and $\left.\mathbf{a}_{\text {Cor }}\right)$ are 'quasi-steady' in that they depend on the instantaneous value of the angular velocity $\boldsymbol{\Omega}$ of the wing (Eqns 12 and 13), in contrast to the angular acceleration ( $\mathbf{a}_{\text {ang }}$ ), which depends on the rate of change of angular velocity $\dot{\boldsymbol{\Omega}}$ (Eqn 11). These rotational accelerations arise in the fluid because the 'fluid particles' on the wing surface are forced to rotate with the same angular velocity and angular acceleration as the wing. The angular velocity and acceleration acquired by the 'fluid particles' on the surface then diffuse into the flow and form the boundary layer around the wing in which the Coriolis acceleration acts on the fluid when it moves with respect to the rotating wing.

\section{Scaling rotational accelerations due to wing stroke}

We now simplify and interpret the dimensionless scale factors of the rotational accelerations (Eqn 22). Using Eqns 26 and 27, we can calculate the (absolute) time-averaged values for $\Omega$ and $\dot{\Omega}$ based on stroke kinematics:

$$
\begin{gathered}
\Omega=\frac{1}{T} \int_{0}^{T}|\dot{\phi}| \mathrm{d} t=\frac{1}{T} \int_{0}^{T}\left|\Phi_{0} 2 \pi f \cdot \cos (2 \pi f t)\right| \mathrm{d} t=4 \Phi_{0} f, \\
\dot{\Omega}=\frac{1}{T} \int_{0}^{T}|\ddot{\phi}| \mathrm{d} t=2 \pi f \Omega,
\end{gathered}
$$

in which $T$ is the flap period, with $T=1 / f$. The wingtip path is illustrated in Fig. 5. The tip speed consists of a component in the direction of flight $u_{\mathrm{t}}$ and normal to it $u_{\mathrm{n}}$ from which we can calculate the absolute, time-averaged, wingtip speed:

$$
U=\frac{1}{T} \int_{0}^{T}|\mathbf{u}(R)| \mathrm{d} t=\frac{1}{T} \int_{0}^{T} \sqrt{u_{\mathrm{t}}^{2}+u_{\mathrm{n}}^{2}} \mathrm{~d} t .
$$

It is essential that both wing and body speed are included in the average speed $U$, so that it represents the right magnitude and ensures that we can continuously scale the NS equation from fast-forward to hovering flight (Lentink and Gerritsma, 2003). We now insert Eqn 26 into Eqn 32 and calculate the tangential and normal velocity components due to the angle between the stroke plane and direction of flight $\gamma$ (Fig. 4B):

$$
\begin{aligned}
& U= \\
& \frac{1}{T} \int_{0}^{T} \sqrt{\left(U_{\infty}+R \cdot \Phi_{0} 2 \pi f \cdot \cos (2 \pi f t) \cdot \sin (\gamma)\right)^{2}+\left(R \cdot \Phi_{0} 2 \pi f \cdot \cos (2 \pi f t) \cdot \cos (\gamma)\right)^{2}} \mathrm{~d} t,
\end{aligned}
$$

in which $U_{\infty}$ is the body speed. We found in an earlier study (Lentink and Gerritsma, 2003) that this equation can be approximated with an error less than $5 \%$ for $\gamma=0^{\circ}$ and $0^{\circ} \leq \arctan (J) \leq 90^{\circ}$, in which $J=U_{\infty} / 4 \Phi_{0} R f$ is the advance ratio:

$$
U \approx \sqrt{U_{\infty}^{2}+\left(4 \Phi_{0} R f\right)^{2}}
$$

This approximation is derived such that it is exact for both hovering $\left(U_{\infty}=0\right)$ and non-flapping flight $(f=0)$. In Fig. 6 we show that this approximation also holds for $0^{\circ} \leq \gamma \leq 90^{\circ}$ and we will therefore incorporate it into our model. We now substitute the expressions for $\Omega$ (Eqn 30), $\dot{\Omega}$ (Eqn 31) and $U$ (Eqn 34) into the scaled NS equation (Eqn 22) and obtain an easier to interpret dimensionless 

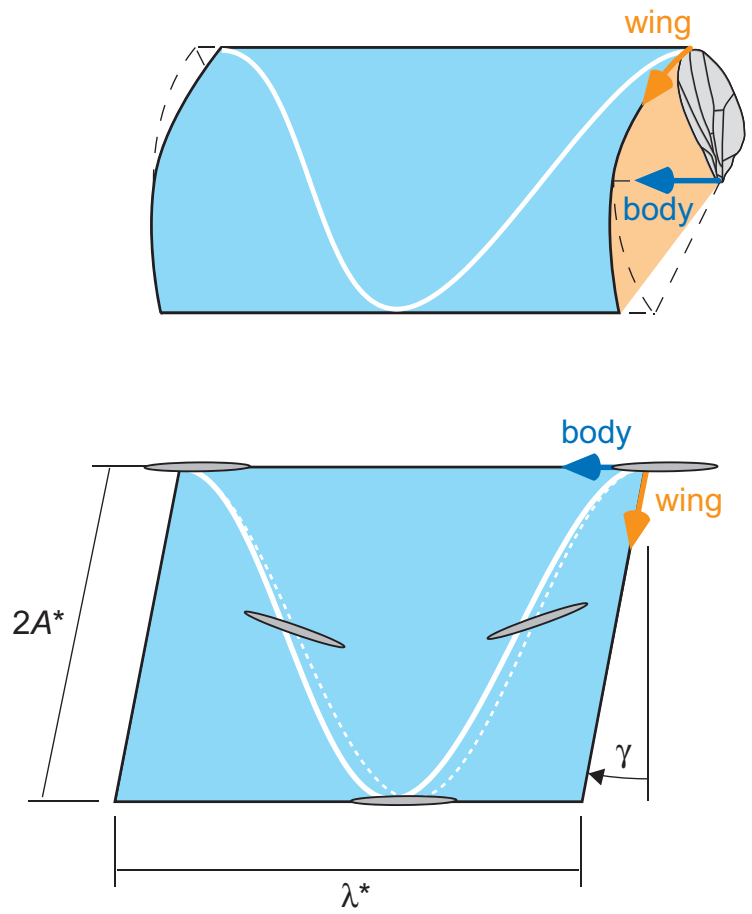

Fig. 5. Flapping wing in forward flight. Graphical representation of the dimensionless numbers in the NS equation that describe the wingtip kinematics; $A^{*}$ the dimensionless stroke amplitude and $\lambda^{*}$ the dimensionless wavelength. The body and wingtip speed are indicated with blue and orange vectors, respectively. The dashed line is the wingtip path for $\gamma=0^{\circ}$ (fast forward or climbing flight). This figure holds for arbitrary flight direction with respect to gravity.

NS equation for a flapping fly wing in forward flight (see Appendix II):

$$
\begin{aligned}
\frac{D \mathbf{u}}{D t}+\frac{1}{J^{2}+1}\left(\frac{1}{A^{*}} \cdot \dot{\boldsymbol{\Omega}} \times \mathbf{r}+\frac{1}{A R_{\mathrm{s}}} \cdot \boldsymbol{\Omega} \times(\boldsymbol{\Omega} \times \mathbf{r})+\right. \\
\left.\frac{1}{A R_{\mathrm{s}}} \cdot 2 \boldsymbol{\Omega} \times \mathbf{u}\right)=-E u \cdot \nabla p+\frac{1}{R e} \cdot \nabla^{2} \mathbf{u},
\end{aligned}
$$

in which $J$ is the advance ratio, $A^{*}$ the dimensionless stroke amplitude of the wing, $A R_{\mathrm{S}}$ the single-wing aspect ratio, $E u$ the Euler number, which is irrelevant for incompressible flow around insects (White, 1991), and Re the Reynolds number. The definitions of the dimensionless numbers in Eqn 35 are as follows:

$$
\begin{gathered}
J=U_{\infty} / 4 \Phi_{0} R f=\lambda^{*} / 4 A^{*}, \\
A^{*}=\frac{\Phi_{0} R}{c}, \\
A R_{\mathrm{s}}=\frac{R}{c}, \\
R e=\sqrt{\left(\frac{U_{\infty} c}{v}\right)^{2}+\left(\frac{4 \Phi_{0} R f_{c}}{v}\right)^{2}}=\sqrt{R e_{\mathrm{b}}^{2}+R e_{\mathrm{s}}^{2}}=\sqrt{J^{2}+1} \cdot R e_{\mathrm{s}} .
\end{gathered}
$$

Note that the Reynolds number consists of two components; the first is due to body speed, $R e_{\mathrm{b}}$, and the second due to wing stroke, $R e_{\mathrm{s}}$.

\section{Graphical representation of dimensionless numbers}

To better understand how the dimensionless numbers in the NS equation of a flapping wing relate to wing kinematics and morphology we represent them graphically. The advance ratio (Eqn 36) is equal to dimensionless wavelength $\lambda^{*}=U_{\infty} / f_{c}$ divided by the total wingtip excursion in the stroke plane $4 A^{*}$ (Fig. 5). The fly hovers for $J=0$ and flies forward (or descends) in an arbitrary direction when $J>0$. For $\gamma=0^{\circ}$, which approximates fast forward and climbing flight, the advance ratio is a direct measure of the average pitch of a flapping wing. The average pitch is a measure of the average induced angle of attack of the flapping wing (Fig. 7) and determines together with the geometric angle of attack amplitude (with amplitude $\alpha_{0}$ ) the average effective angle of attack amplitude (Fig. 7). The effective angle of attack amplitude modulates wing lift and drag. For $0^{\circ}<\gamma \leq 90^{\circ}$ (Fig. 5), the geometric interpretation of $J$ is gradually modified, $\gamma=90^{\circ}$ being the extreme case. This case is relevant for slow hovering (Dickson and Dickinson, 2004); under such conditions the advance ratio also measures how much the fly moves forward along its flight path compared with its total stroke length. The average induced angle of attack is, however, zero, because it is proportional to $\cos (\gamma)$.

The geometric representation of the dimensionless amplitude $A^{*}$ (Eqn 37) is shown in Fig. 5 and its geometric interpretation is simple. An equivalent dimensionless total amplitude $\Lambda=2 A^{*}$, has been defined by Ellington (Ellington 1984). The geometric interpretation of the single-wing aspect ratio (Eqn 38) is also straightforward and can be inferred from Fig. 3B by noting $A R_{\mathrm{s}}=R / c=R b_{\mathrm{s}} / S$ (in which $b_{\mathrm{s}}$ is the single-wing span). Finally, $R e$ can be interpreted as the ratio of convective versus diffusive transport length for a fixed time interval. It measures how strongly the velocity boundary condition at the wing surface is diffused into the flow and is a measure of boundary layer thickness (e.g. Schlichting, 1979; Tennekes and Lumley, 1983) (Fig. 2).

How do scale factors of the rotational accelerations in Eqn 35 behave? The angular acceleration scales with $1 /\left(J^{2}+1\right) A^{*}$, which increases for decreasing $A^{*}$ at constant $J$ and increases for decreasing $J$ at constant $A^{*}$. When $A^{*}=0$ a careful analysis of the product is needed, which shows that it will become zero (non-singular) provided that $U \neq 0$; there is flow. The analysis holds for the subsequent terms discussed below; they are also non-singular provided that $U \neq 0$. The centripetal and Coriolis accelerations scale with $1 /\left(J^{2}+1\right) A R_{\mathrm{S}}$, which increases for decreasing $A R_{\mathrm{S}}$ at constant $J$ and increases for decreasing $J$ at constant $A R_{\mathrm{S}}$.

\section{Hovering flight}

When insects fly slowly at advance ratios less than 0.1 they hover according to the definition of Ellington (Ellington, 1984). This notion is confirmed by calculating the scale factors in Eqn 35. The effect of forward flight is scaled by the inverse of $J^{2}+1$ for Coriolis, centripetal and angular acceleration, which makes them insensitive to low $J$ values. Evaluating $J=0$ and 0.1 yields two scale factors that differ by only $1 \%$. For such low values of $J$ we can simplify Eqn 35 into:

$$
\begin{aligned}
\frac{D \mathbf{u}}{D t}+\frac{1}{A^{*}} \cdot \dot{\boldsymbol{\Omega}} \times \mathbf{r}+\frac{1}{A R_{\mathrm{s}}} \cdot \boldsymbol{\Omega} \times(\boldsymbol{\Omega} \times \mathbf{r})+ & \frac{1}{A R_{s}} \cdot 2 \boldsymbol{\Omega} \times \mathbf{u}= \\
& -E u \cdot \nabla p+\frac{1}{R e} \cdot \nabla^{2} \mathbf{u} .
\end{aligned}
$$

Perhaps this equation will remain a good approximation for even higher $J$ values. Many insects seem to perform such flights during 


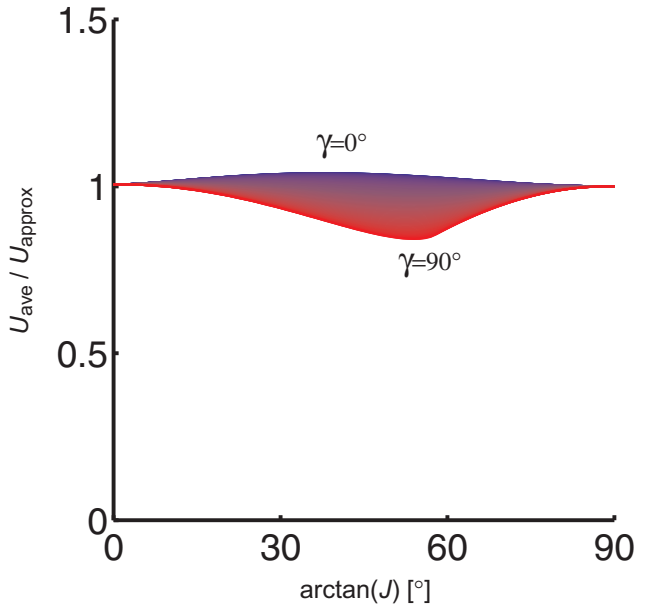

Fig. 6. The absolute average of the wingtip velocity $\left(U_{\text {ave }}\right)$ can be approximated well $\left(U_{\text {approx }}\right)$ within the same order of magnitude using Eqn 34 for any combination of $\gamma$ and $J$ (advance ratio).

vertical take-off and landing and slow hovering flights (Ellington, 1984), which makes Eqn 40 particularly useful.

\section{From flapping to spinning and translating fly wings}

The aerodynamics of flapping and spinning fly wings share important traits at zero advance ratio. Dickinson and co-workers (Dickinson et al., 1999) showed that a 'quasi-steady' aerodynamic model of a flapping fruit fly wing can predict the majority of the lift generated, based on the lift that the same wing generates when it simply spins. Ellington and collaborators showed for hawkmoths that both flapping and spinning wings generate a stable leading edge vortex (LEV) (Ellington et al., 1996; Usherwood and Ellington, 2002). These findings suggest that the aerodynamics of flapping and spinning insect wings could be similar to the aerodynamics of propellers (Himmelskamp, 1947) and wind turbines (e.g. Dumitrescu and Cardos, 2003; Tangler, 2004). Hence we further simplify Eqn 35 for an insect wing spinning at constant angular velocity $(\dot{\phi}=0)$ at a constant geometric angle of attack $(\dot{\alpha}=\ddot{\alpha}=0)$ :

$$
\begin{aligned}
& \frac{D \mathbf{u}}{D t}+\frac{1}{J^{2}+1}\left(\frac{1}{A R_{\mathrm{s}}} \cdot \boldsymbol{\Omega} \times(\boldsymbol{\Omega} \times \mathbf{r})+\frac{1}{A R_{\mathrm{s}}} \cdot 2 \boldsymbol{\Omega} \times \mathbf{u}\right)= \\
& \quad-E u \cdot \nabla p+\frac{1}{R e} \cdot \nabla^{2} \mathbf{u} .
\end{aligned}
$$

Because angular velocity is now constant we need to rewrite the definition of advance ratio as:

$$
J=U_{\infty} / 2 \pi R f,
$$

and the Reynolds number as:

$$
R e=\sqrt{\left(\frac{U_{\infty} c}{v}\right)^{2}+\left(\frac{2 \pi R f c}{v}\right)^{2}}=\sqrt{R e_{\mathrm{b}}^{2}+R e_{\mathrm{s}}^{2}}=\sqrt{J^{2}+1} \cdot R e_{\mathrm{s}} .
$$

The corresponding graphical interpretation of the dimensionless numbers is illustrated in Fig. 8. During one full period the wingtip has rotated over a dimensionless circular distance equal to $D^{*}=2 \pi R / c$ and has moved forward through the air over a linear distance of $s^{*}=U_{\infty} / c f$.

The single difference between propeller and turbine kinematics is that propellers operate at positive angles of attack generating
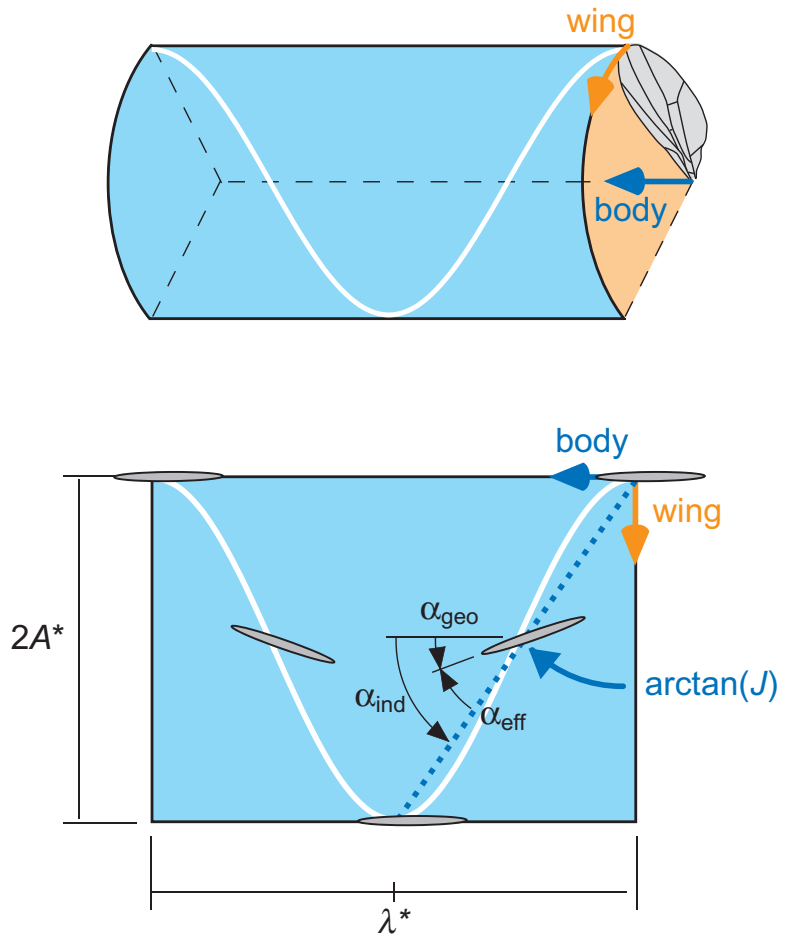

Fig. 7. Graphical representation of $A^{*}$ and $\lambda^{*}$ for $\gamma=0^{\circ}$; fast forward or climbing flight. Under these conditions $\arctan (1 / \mathcal{J})$ is the average induced angle of attack $\alpha_{\text {ind }}$ of the flapping wing. The maximum induced angle of attack amplitude at midstroke can be calculated using $\arctan (\pi / 2 \mathcal{L})$ (Lentink et al., 2008). The effective angle of attack of the wing $\alpha_{\text {eff }}$ is equal to the induced angle of attack $\alpha_{\text {ind }}$ minus the geometric angle of attack $\alpha_{\text {geo }}$.

forward pointing lift, which costs power, while turbines operate at negative angles of attack, which results in backward pointing lift and allows for the harvesting of power from wind (and water currents). The comparison of Eqn 41 and 35 shows that the dimensionless numbers and accelerations involved in the aerodynamics of flappers, propellers and turbines are indeed similar, provided that unsteady effects measured by $A^{*}$ do not dominate over 'quasi-steady' rotational effects measured by $A R_{\mathrm{S}}$. By noting that $A^{*}=\Phi_{0} A R_{\mathrm{s}}$ (combining Eqns 37 and 38) for a fly wing and that $\Phi_{0}$ is typically close to one for insects (Ellington, 1984), we find that unsteady accelerations measured by $1 / A^{*}$ and 'quasi-steady' accelerations measured by $1 / A R_{\mathrm{S}}$ are of the same magnitude. This might explain the physical analogy between flapping and spinning insect wings and, possibly, propellers and turbines that operate at much higher Reynolds numbers. Propellers that operate at zero advance ratio, $J=0$, operate under hover conditions, such as hovering insects, which further simplifies Eqn 41 .

Although flies are not known to glide, dragonflies and many birds and bats do. When animals glide the transformed NS equation that describes the aerodynamics of their translating wings has the same form as those for airplane wings (Fig. 2), which is obtained by setting $\mathbf{\Omega}=\mathbf{0}$ in Eqn 41.

\section{D pitch and heave wing kinematics}

There are a large number of insect flight models in the literature that range from fully 3D wing kinematics (e.g. Ellington et al., 1996; Dickinson et al., 1999) to 2D pitch and heave kinematics [e.g. Dickinson, who used end-baffles to make the flow quasi-2D 

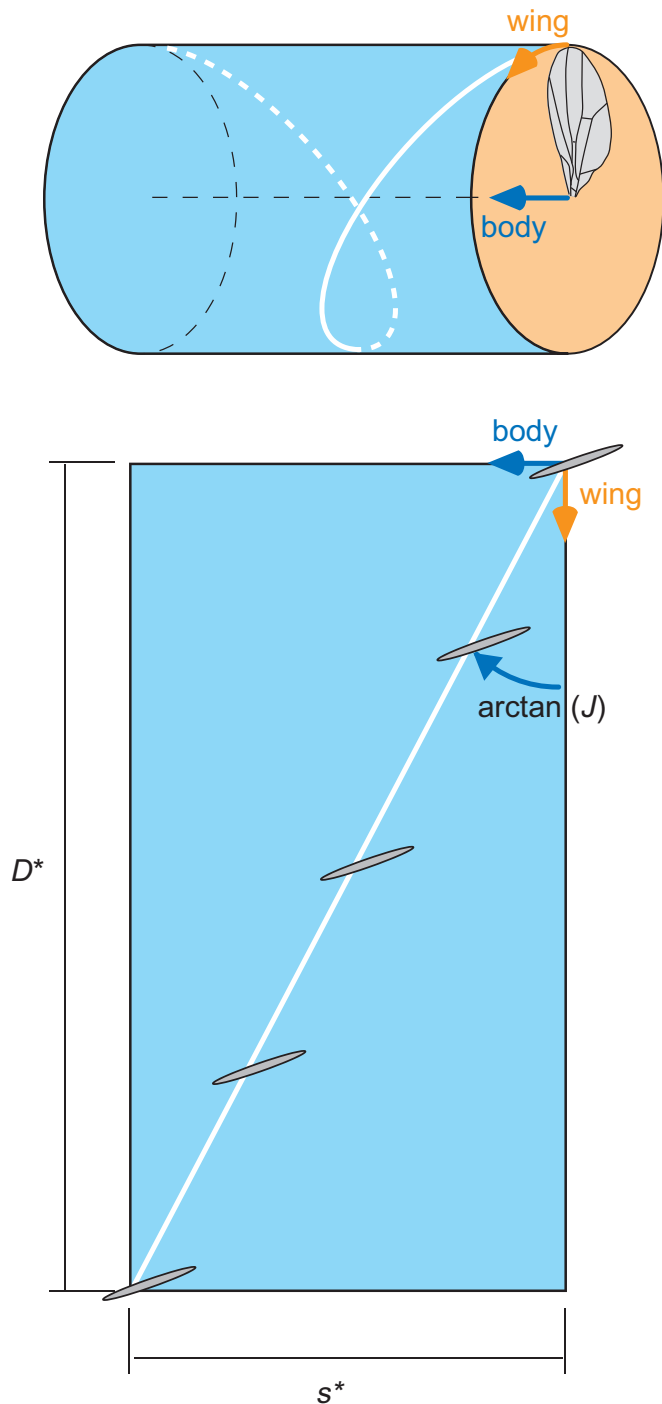

Fig. 8. Propeller in forward flight. Graphical representation of the dimensionless numbers in the NS equation that describe the wingtip kinematics; $D^{*}$ and $s^{*}$. Note that the induced angle of attack is again $\arctan (1 / \mathcal{J})$.

(Dickinson, 1994)]. A further simplification is to consider a wing cross-section; the airfoil ( $z$-coordinate constant) (e.g. Wang, 2000b; Lentink et al., 2008). Although 2D studies are more restrictive, they have provided valuable insight into insect wing performance. Here we focus on a 3D wing that pitches sinusoidally with amplitude $\alpha_{0}$ and heaves sinusoidally with amplitude $A$. Because the sinusoidal heave kinematics results in a linear acceleration of the wing, this linear acceleration term $\mathbf{a}_{\text {wing }}$ must be added to Eqn 10. The (pitch and) heave kinematics is identical to the 2D flattened stroke plane shown in Fig. 5 (lower panel). The wing stroke acceleration is derived similarly to Eqns 27 and 29:

$$
\ddot{S}_{\text {wing }}=-A(2 \pi f)^{2} \sin (2 \pi f t),
$$

in which $\ddot{s}_{\text {wing }}$ is the stroke position of the wing in the stroke plane. Calculation of the corresponding (absolute) time-averaged acceleration $\dot{U}_{\text {wing }}$ is analogous to Eqns 30 and 31 :

$$
\dot{U}_{\text {wing }}=2 \pi f \cdot 4 \mathrm{~A} f \text {. }
$$

The resulting scaled order of magnitude of the wing's heave acceleration $\mathbf{a}_{\text {wing }}$ is:

$$
\frac{\dot{U}_{\text {wing }} c}{U^{2}}=\frac{1}{J^{2}+1} \cdot \frac{1}{A^{*}} .
$$

This scale factor multiplied by the dimensionless wing acceleration $\mathbf{a}_{\text {wing }}$ is inserted into the dimensionless NS equation Eqn 22 of the pitching and heaving foil. The relevant radius now becomes the pitch radius, which scales with $c$ instead of $R$ (Appendix II):

$$
\begin{aligned}
& \frac{D \mathbf{u}}{D t}+\frac{1}{J^{2}+1} \cdot\left(\frac{1}{A^{*}} \cdot \mathbf{a}_{\text {wing }}+\frac{\alpha_{0}}{A^{*^{2}}} \cdot \dot{\mathbf{\Omega}} \times \mathbf{r}+\frac{\alpha_{0}^{2}}{A^{*^{2}}} \cdot \mathbf{\Omega} \times\right. \\
&\left.(\mathbf{\Omega} \times \mathbf{r})+\frac{\alpha_{0}}{A^{*}} \cdot 2 \boldsymbol{\Omega} \times \mathbf{u}\right)=-E u \cdot \nabla p+\frac{1}{R e} \cdot \nabla^{2} \mathbf{u} .
\end{aligned}
$$

with the following expressions for advance ratio, which are similar to Eqn 36:

$$
J=U_{\infty} / 4 A f=\lambda * / 4 A^{*} .
$$

Note that the acceleration terms in Eqn 47 are much simplified compared with 3D kinematics because many vector components are zero:

$$
\mathbf{a}_{\text {wing }}=\left[\begin{array}{c}
\ddot{x}_{\text {wing }} \\
\ddot{y}_{\text {wing }} \\
0
\end{array}\right], \dot{\mathbf{\Omega}}=\left[\begin{array}{c}
0 \\
0 \\
\ddot{\alpha}
\end{array}\right], \mathbf{\Omega}=\left[\begin{array}{c}
0 \\
0 \\
\dot{\alpha}
\end{array}\right], \mathbf{r}=\left[\begin{array}{c}
x \\
y \\
z
\end{array}\right] .
$$

From inspecting Eqn 47 we conclude that the rotation corresponding with the angle of attack induces rotational accelerations in the plane of the airfoil, which are proportional to the geometric angle of attack amplitude. These rotational acceleration components are also present in Eqn 35, but are dominated by those due to the wing stroke (its propeller-like swing). The rotational accelerations due to stroke can have a component normal to the $z$-plane which confines the wing's airfoil. The relative magnitude of the accelerations for $A^{*}=0$ can again be inferred correctly by either reformulating the scale term as a whole or calculating the limit value, because $J$ also depends on $A^{*}$ (Eqn 48). The expression for the Reynolds number is similar to Eqn 39; it can be obtained by inserting $A=\Phi_{0} R$.

\section{Simple vibrating fly wings}

Some insect wing models are even simpler and just consist of a heaving (vibrating) foil at constant geometric angle of attack ( $\alpha_{0}=$ constant) (e.g. Wang, 2000b; Lentink and Gerritsma, 2003):

$$
\frac{D \mathbf{u}}{D t}+\frac{1}{J^{2}+1} \frac{1}{A^{*}} \cdot \mathbf{a}_{\mathrm{wing}}=-E u \cdot \nabla p+\frac{1}{R e} \cdot \nabla^{2} \mathbf{u} .
$$

Using Eqn 48 we can simplify $\left(J^{2}+1\right) A^{*}$ to:

$$
\frac{1}{4^{2} A^{*}}\left(\lambda^{* 2}+4^{2} A^{* 2}\right)
$$

which relates to the synchronization bands Williamson and Roshko (Williamson and Roshko, 1988) found in experiments with a vibrating cylinder in the parameter space spanned by dimensionless wavelength ( $X$-axis) and amplitude ( $Y$-axis). In this elliptically shaped synchronization band the vortex wake synchronizes with the vibrating cylinder. This occurs when the cylinder effectively vibrates at a multiple of the natural von Kármán vortex shedding wavelength $\lambda_{0}$ of the cylinder at rest: $n \lambda_{0} *=\sqrt{ }\left(\lambda^{* 2}+4^{2} A^{* 2}\right)$ for $n=1$ and 3 (Lentink, 2003; Ponta and Aref, 2005). The present derivation 


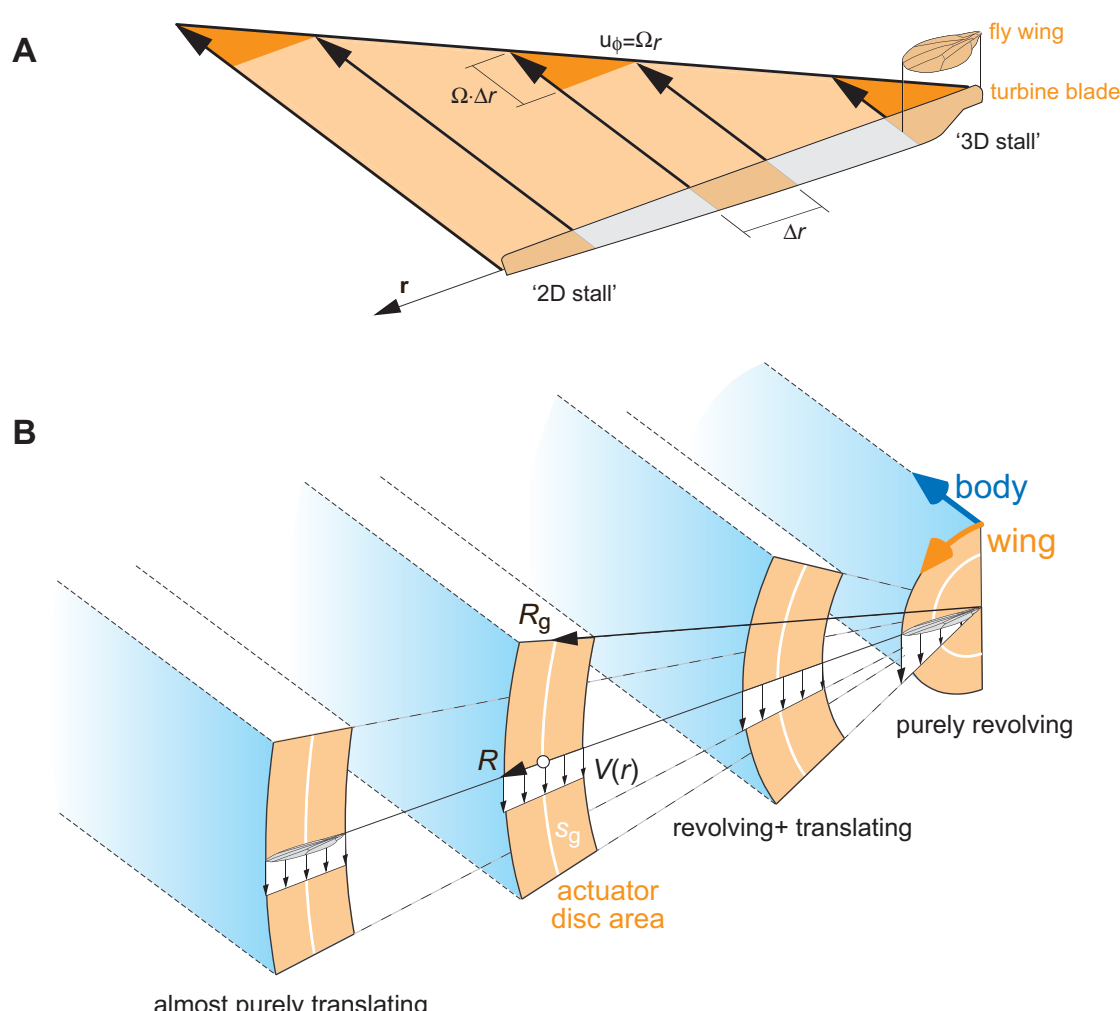

Fig. 9. Is there an analogy between the influence of rotational accelerations on the stall characteristics of wind turbines and flapping fly wings? (A) Slender wings, such as wind turbine blades, have been found to possess distinctly different stall mechanisms near the root, for $r / c<3$ where they stall locally ' $3 \mathrm{D}$ ', and near the tip, where they stall locally '2D'. Fly wings are much less slender than wind turbine blades, therefore their whole wing is 'submerged' in the region of '3D stall'. Clearly the circular path (in the stroke plane) of outward wing sections becomes progressively less curved compared with chord length. We illustrated how the local 'rotational component' of the radial speed component $\boldsymbol{\Omega} \cdot \Delta r$ becomes less and less important towards the tip compared with the 'linear component' of radial speed $\Omega r$. By taking $\Delta r=c$ we find that the ratio of 'linear component' over 'rotational component' of radial speed is exactly $r / c$. (B) Based on the observations in A we designed flapping wing kinematics that allow us to change the relative importance of rotational accelerations $R / c$ for a fly wing, which has a constant geometric slenderness. We achieved such a gradual transition from a linear velocity distribution to a constant velocity over the wing by gradually sticking the fly wing further out on an extension rod between the wing and its joint. In this we keep the stroke amplitude 'actuator disc' areas swept by the wing in the stroke plane constant (orange) while it travels forward (blue). It can be demonstrated that a constant actuator disc area corresponds with a nearly constant stroke amplitude along the path $s_{\mathrm{g}}$ at the radius of gyration $R_{\mathrm{g}}$. The relative curvature of the path of the wing sections with respect to its chord length is again measured by $R / c$, the single-wing aspect ratio. Graphically we can directly infer that the paths traveled at more outward radii are much less curved and therefore the corresponding 3D rotational accelerations will diminish.

shows that this relationship can be linked to the scale factor that represents the linear acceleration of the vibrating cylinder in the NS equation (Eqn 53). Whether vortex wake synchronization bands also occur for flapping wings is unclear (Lentink et al., 2008).

\section{DISCUSSION}

We derived a dimensionless form of NS equations for a 3D flapping fly wing to identify the dimensionless numbers that scale the underling physical mechanisms. This derivation shows that flapping wings induce three rotational accelerations: angular, centripetal and Coriolis in the air near to the wing's surface, which diffuse into the boundary layer of the wing. Next we simplified these equations incrementally using increasingly more restrictive assumptions. These simplifications allow us to easily interpret the dimensionless numbers geometrically for conditions that approximate both forward flight and hovering. In subsequent steps we derived the NS equation for spinning and translating 3D fly wings and for 2D flapping and vibrating wings.

Dimensionless template for parametric flapping wing studies The dimensionless numbers that scale the NS equation facilitate the design of the parametric space in which one can systematically investigate the fluid dynamics of flapping wings. These numbers are of additional importance to other kinematic parameters of the wing such as angle of attack amplitude $\alpha_{0}$ and the angle between the normal vector of the average stroke plane and the direction of flight $\gamma$. When the most general conditions hold (Eqn 22), we find the following relevant scale factors:

$$
\frac{\dot{\Omega} R c}{U^{2}}=\frac{1}{C_{\text {ang }}},
$$

in which $C_{\text {ang }}$ is the angular acceleration number;

$$
\frac{\Omega^{2} R c}{U^{2}}=\frac{1}{C_{\mathrm{cen}}}
$$

in which $C_{\text {cen }}$ is the centripetal acceleration number;

$$
\frac{\Omega^{2} R c}{U^{2}}=\frac{1}{R o},
$$

in which $R o$ is the Rossby number;

$$
\frac{\mu}{\rho U c}=\frac{1}{R e},
$$


in which $R e$ is the Reynolds number. Using this notation we are able to accommodate elegantly the definition of both Reynolds (Reynolds, 1883) and Rossby numbers (Rossby, 1936) that already exist in the biological and rotational fluid dynamics field. Three of these terms are relatively new in the biological fluid mechanics field: (1) the angular acceleration number measures the unsteadiness of the flow induced by the rotational acceleration of the wing; (2) the centripetal acceleration number measures the centripetal acceleration due to the wing's rotation, and (3) the Rossby number measures the Coriolis acceleration induced by the relative motion of fluid with respect to the rotating wing. All three numbers represent the relative magnitude of the corresponding acceleration with respect to the convective flow acceleration. Further, all these rotational accelerations diminish for large values of the dimensionless numbers that represent them. For animals we find that $C_{\text {cen }}$ and $R o$ are typically identical, because this case corresponds with high locomotory performance (see Appendix I). But in general, e.g. some engineering problems or special maneuvers in which high $\gamma$ occur, they are not identical and therefore we distinguish between them here (Eqns 56 and 57). Although these numbers can be readily calculated they do not give clear insight into how they represent wing kinematics and morphology, which can be critical for the design of a careful parametric study of flapping wing aerodynamics. Therefore we developed a geometric framework to provide insight into the dimensionless numbers that describe the aerodynamics of the flapping wings of a fly flying at constant velocity. Within this framework we assumed sinusoidal stroke and angle of attack kinematics and zero wing deviation. Comparing Eqn 22 and Eqn 35 we find the following geometric interpretation for the dimensionless numbers $C_{\text {ang }}, C_{\text {cen }}$ and $R o$ :

$$
\begin{aligned}
& C_{\text {ang }}=\left(J^{2}+1\right) A^{*}, \\
& C_{\text {cen }}=\left(J^{2}+1\right) A R_{\mathrm{s}}, \\
& R o=\left(J^{2}+1\right) A R_{\mathrm{s}} .
\end{aligned}
$$

Whilst $J$ and $A^{*}$ are measures of the wing's kinematics, $A R_{\mathrm{S}}$ is a measure of single-wing morphology. Again we note that the effect of large values of these dimensionless numbers is that it diminishes the corresponding accelerations. The effect of forward flight $(J>0)$ is, therefore, that it reduces the rotational accelerations. The rotational accelerations increase for smaller stroke amplitudes and single-wing aspect ratios at constant advance ratio. The extreme case is hovering flight $(J=0)$ for which the rotational accelerations are maximal. Using the dimensionless scale variables $J, A^{*}$ and $A R_{\mathrm{S}}$ we can now systematically vary the influence of rotational accelerations in parametric studies of the aerodynamics of fly wings in forward and hovering flight. There are, however, alternative ways to combine or split up the principal dimensionless numbers $C_{\mathrm{ang}}$, $C_{\text {cen }}$ and $R o$ into other dimensionless factors, because they all depend on the same set of scale variables (length, time and mass). Alternatives are, for example, factors that more intuitively represent ratios of force or time scales. We chose to build up our three principal dimensionless numbers (Eqns 59-61) such that they correspond with the easy to interpret geometrical scales presented in Figs 5, 7 and 8. This approach facilitates an intuitive design of numerical and experimental studies of flapping wings with a direct link to the NS equations.

\section{Comparing 3D and 2D wing kinematics}

How important are rotational accelerations for understanding the aerodynamics of a fly? Here we present an alternative approach to compare $2 \mathrm{D}$ and $3 \mathrm{D}$ stroke kinematics, which proved to be pivotal for designing our experiment to test how important rotational accelerations (due to stroke) are for the stability of a fly's LEV (Lentink and Dickinson, 2009). This vortex allows the fly to generate high lift with its wing at angles of attack at which helicopter blades and airplane wings stall. There exists, however, an intriguing parallel between the lift augmentation due to the presence of a stable LEV on a fly wing and the lift augmentation found near the hub of wind turbine blades. Such blades are said to undergo '3D stall' or 'stall delay' near their hub, which increases lift, whereas they undergo '2D stall' near the blade tip, which decreases lift (e.g. Tangler, 2004) (Fig.9A). For wind turbines 3D stall is not observed beyond 'local aspect ratios' of three $(r / c>3$, in which $r$ is the local radius; see Fig. 9A) (e.g. Tangler, 2004). Three is approximately the value of a fruit fly's wing aspect ratio (Fig. 9), which might explain why flapping and spinning fly wings do not seem to stall and generate extraordinary high lift like wind turbines and propellers (Himmelskamp, 1947) do near their hub. To gain insight into the possible effect of rotational accelerations we gradually transform rotational stroke kinematics into translational stroke kinematics (Fig. 9B). We do this by letting $\Phi_{0} \rightarrow 0$ and $R \rightarrow \infty$ such that the wing amplitude $\Phi_{0} R=A$ remains constant within the limit. In doing so we do not change the wing's geometry, we simply place the same wing farther outward as shown in Fig. 9. Because the wing's radial distance from the center of rotation $R$ goes to infinity $(R \rightarrow \infty)$, the single-wing aspect ratio based on this radial distance $A R_{\mathrm{s}}=R / c$ also goes to infinity. The NS equations of a flapping (Eqn 35) and spinning wing (Eqn 41) show that the centripetal and Coriolis acceleration go down with aspect ratio. We have performed exactly this experiment to show that rotational accelerations mediate LEV stability in hovering insect flight (Lentink and Dickinson, 2008). The similar importance of rotational accelerations (e.g. for LEV stability) for flapping and spinning wings are confined to the midstroke phase of the flapping cycle when the flapping wing revolves with a propeller-like swing at nearly constant angular acceleration. This phase corresponds with the maximum dynamic pressure due to wing stroke and therefore dominates lift production for most insects. In practice this analogy is complicated by the effects of significant stroke acceleration, wing deviation and wing rotation at the beginning and end of the stroke (e.g. Dickinson et al., 1999; Altshuler et al., 2005). These significant complicating factors do not, however, modify the analogy between the flow physics and forces we (Lentink and Dickinson, 2009) and others (e.g. Dickinson et al., 1999; Usherwood and Ellington, 2002) found at midstroke for flapping and spinning insect wings.

\section{Rotational accelerations due to wing stroke versus angle of attack kinematics}

The angular velocity of flapping wings consists of two significant components. (1) Angular velocity due to wing stroke and (2) angular velocity due to geometric angle of attack variation. Angular velocity due to wing stroke is approximately maximal at midstroke, while angular velocity due to wing angle of attack variation is approximately maximal at stroke reversal. The resulting velocity magnitudes are proportional to $\Phi_{0} R f$ for stroke and $\alpha_{0} c f$ for angle of attack. The ratio of the two velocities is $\Phi_{0} R / \alpha_{0} c<R / c$ because $\Phi_{0}<\alpha_{0}$ for most insects. For aspect ratios significantly larger than one we can assume, therefore, that rotational accelerations due to stroke dominate those due to angle of attack variation. One can test experimentally whether this holds by doing the experiments with 
and without rotational stroke kinematics (reciprocating revolving versus reciprocating translating wings) for all relevant angle of attack amplitudes, which lie in the range $0^{\circ} \leq \alpha_{0} \leq 90^{\circ}$ (Lentink and Dickinson, 2009).

\section{Scaling the NS equation more accurately}

In some fly wing experiments more accurate dimensionless numbers are needed. For example in a careful comparison of aerodynamic forces that result from different, but related, wing kinematics. In such cases one needs to calculate the velocity and length scales more accurately by using a blade element method. For such detailed lift and drag studies, the wing radius of gyration should be chosen as the radial length scale and the accompanying velocity at the radius of gyration should be chosen as the velocity scale in the NS equation. Calculating the radius of gyration is straightforward for hovering insects (Weis-Fogh, 1973; Ellington, 1984):

$$
R_{\mathrm{g}}=\sqrt{\frac{1}{S} \int_{0}^{R} r^{2} c(r) \mathrm{d} r},
$$

in which $R_{\mathrm{g}}$ is the radius of gyration and $S$ the single-wing area. For forward flight conditions this computation gets increasingly more complicated (e.g. Dickson and Dickinson, 2004). In practice it is therefore more convenient to consider the wing radius instead of the radius of gyration, because this can be derived much more easily from lab and field data. The values of wing radius and wing speed (due to stroke) at the radius of gyration are roughly half the value calculated at the wingtip, because $R_{\mathrm{g}} / R<0.5$ (Ellington, 1984). This estimate helps in evaluating whether more precise values would change the conclusions using 'back of the envelope' calculations. Finally we note that we approximate $\pi / 2 \approx 1$ in Appendix II; this ratio could be approximated more accurately by $\pi / 2 \approx 1.5$ or a better approximation if needed (e.g. when solving the scaled NS equations numerically through CFD).

\section{Relationship between existing and present dimensionless numbers}

Some of the dimensionless numbers that we derived here can be related to existing dimensionless numbers. The advance ratio $J$ is already in use (Ellington, 1984). It is equivalent to the inverse of the amplitude-based Strouhal number as discussed in the Introduction. We prefer the advance ratio because it is readily interpretable geometrically and commonly used as such in aeronautics and biological fluid dynamics. Further, the Strouhal number is perhaps best reserved for its original purpose characterizing natural shedding frequencies. The dimensionless amplitude $A^{*}$ is a normal dimensionless variable, $2 A^{*}$ represents the total dimensionless amplitude $\Lambda$ introduced by Ellington (Ellington, 1984); we prefer $A^{*}$, because it represents the mathematical definition of amplitude. We further prefer the dimensionless wavelength $\lambda^{*}$ over the inverse, the reduced frequency $k$, because $\lambda *$ is a length scale ratio that can easily be drawn and interpreted graphically, e.g. Fig. 5, while the time scale ratio $k$ cannot. The importance of the dimensionless single-wing aspect ratio for calculating rotational accelerations is new in the field of insect flight. The corresponding Rossby number is, however, commonly used in the analyses of rotating fluids (Rossby, 1936; Vanyo, 1993; Greitzer et al., 2004; Tritton, 2005). The inverse of the single-wing aspect ratio, $c / r$, is in use in the wind turbine literature (e.g. Lindenburg, 2004), but we prefer $r / c$ because it corresponds to the single-wing aspect ratio which is easier to interpret geometrically for animal flight, and because $r / c$ corresponds with the definition of Rossby number, which is in use in the much more elaborate literature on rotational flows (compared with wind turbines). Finally, there is the Reynolds number; our definition has the advantage that it works continuously from hovering flight to fast forward flight (Eqn 39) (Lentink and Gerritsma, 2003).

Application of dimensionless numbers in wing and fin studies Our derivation of the dimensionless NS equation for flapping, revolving and translating fly wings and airfoils represent the various $3 \mathrm{D}$ and 2D insect flight models in the literature; from 3D flapping wings to $2 \mathrm{D}$ vibrating wings. By comparing Eqn 35 for a 3D flapping wing and Eqn 47 for a 2D flapping wing we conclude that the significant rotational accelerations due to the flapping motion in 3D fly aerodynamics are neglected in $2 \mathrm{D}$ models. The possible importance of such differences is amplified by the experimental observation that 3D fly wings that either spin (e.g. Usherwood and Ellington, 2002) or flap (e.g. Birch et al., 2004; Ellington et al., 1996) around their base generate a stable LEV, while flapping 2D airfoils (e.g. Dickinson and Goetz, 1993; Dickinson, 1994; Lentink et al., 2008) do not. We further note that 2D vibrating insect wing models neglect all rotational accelerations (e.g. Wang, 2000b; Lentink and Gerritsma, 2003). The above insect flight models therefore increasingly incorporate the rotational accelerations induced in the flow due to the rotational kinematics of the wing motion.

2D and 3D models similar to those of insect flight have also been used to study the aerodynamics of birds (Hubel, 2006) and the hydrodynamics of the fins of fish (Triantafyllou et al., 1993; Bandyopadhyay et al., 2008). The dimensionless NS equation we derived for a flapping fly wing also represents such models, provided that care is taken that the assumptions used to derive the various equations hold. For completeness we repeat three non-trivial assumptions: (1) the fluid behaves in a fully Newtonian way like water and air, (2) the fluid does not cavitate, and (3) the amplitudes of undulations in the body are small compared with stroke amplitude. The flow can, however, be turbulent.

Finally we note that even the abstract problem of spinning fly wings, Eqn 41, is of direct relevance for biological fluid dynamics studies, because autorotating seeds spin with exactly such kinematics as they swirl down to earth (Azuma and Yasuda, 1989). While doing so, autorotating seeds extract energy from the flow very much like wind turbines harvest energy from wind at much higher Reynolds numbers. This intriguing example illustrates that our theoretical approach has the potential to provide a valuable link between the fluid dynamics of translating, rotating and flapping wings and fins in nature and technology.

\section{APPENDIX I}

Contribution of body versus wing velocity to Rossby number In our non-dimensional Eqn 22 and all derived equations thereafter we neglect the influence of the angle between the normal vector of the stroke plane and the body velocity $\gamma$ on the dimensionless number representing the Coriolis acceleration: the Rossby number. Below we argue that the effect of $\gamma$ on Ro is indeed negligible in general for flappers and helicopters that need to operate at reasonable to high efficiency. For this we need to estimate the order of magnitude and direction of the velocity of the average flow close to the wing (above the surface). Based on Eqn 6 and Fig. 4 we estimate:

$$
\mathbf{u}_{\text {loc }} \propto-\left(\mathbf{u}_{\text {body }}+\boldsymbol{\Omega}_{\text {wing }} \times \mathbf{r}\right) .
$$


Using Eqn A1 we can now estimate the order of magnitude of the Coriolis term in Eqn 22 taking the influence of the relative vector directions into account (on which the cross-product depends):

$$
\begin{aligned}
& O\left[\boldsymbol{\Omega}_{\text {wing }} \times \mathbf{u}\right]=O\left[-\boldsymbol{\Omega}_{\text {wing }} \times\left(\mathbf{u}_{\text {body }}+\boldsymbol{\Omega}_{\text {wing }} \times \mathbf{r}\right)\right] \\
& =O\left[-\boldsymbol{\Omega}_{\text {wing }} \times \mathbf{u}_{\text {body }}\right]+O\left[-\boldsymbol{\Omega}_{\text {wing }} \times \boldsymbol{\Omega}_{\text {wing }} \times \mathbf{r}\right] \\
& =\underbrace{\boldsymbol{\Omega}_{\text {wing }} U_{\text {body }} \sin (\boldsymbol{\gamma})}_{\text {I }}+\underbrace{\boldsymbol{\Omega}_{\text {wing }}{ }^{2} R}_{\text {II }} .
\end{aligned}
$$

In this we used Eqns 15-21, and we defined $U_{\text {body }}$ and $\Omega_{\text {wing }}$ as the amplitudes of the corresponding vectors $\mathbf{u}_{\text {body }}$ and $\boldsymbol{\Omega}_{\text {wing. }}$. We now need to determine whether term I or II is dominant in this order of magnitude analysis. For this we calculate the ratio of term I to II, which yields an in-stroke-plane advance ratio $J_{\text {isp }}$ :

$$
J_{\text {isp }}=\frac{\Omega_{\text {wing }} U_{\text {body }} \sin (\gamma)}{\Omega_{\text {wing }}{ }^{2} R}=\frac{U_{\text {body }} \sin (\gamma)}{\Omega_{\text {wing }} R} .
$$

For $J_{\text {isp }}$ close to one or higher there will be flow stagnation or even flow reversal on the wing during either the upstroke or the downstroke, which is highly detrimental to both lift generation and flapping efficiency. This has been found not only in earlier experiments with models of flapping insects wings (Dickson and Dickinson, 2004) but also for helicopters in forward flight and yawed wind turbines experiencing side flow. Based on these findings and assuming animals strive for effective locomotion we conclude that $J_{\text {isp }}$ will typically be smaller than one in animal locomotion, except perhaps during special maneuvers. This yields a negligible influence of $\gamma$ on Ro, which can be seen by substituting Eqn A3 into Eqn A2:

$$
O\left[\boldsymbol{\Omega}_{\text {wing }} \times \mathbf{u}\right]=\Omega_{\text {wing }}^{2} R\left(J_{\text {isp }}+1\right) .
$$

Because this is an order of magnitude analysis we may neglect the contribution of $J_{\text {isp }}$ if it is smaller than one, or of the same order at most, which we argue to be typically the case for animals:

$$
O\left[\boldsymbol{\Omega}_{\text {wing }} \times \mathbf{u}\right]=\Omega_{\text {wing }}^{2} R .
$$

If a more exact approximation is desired Eqn A4 can be used, but in general one can expect this will not be needed because for an order of magnitude analysis $J_{\text {isp }}$ can already be neglected when $O\left[J_{\text {isp }}\right] \leq 1$.

\section{APPENDIX II \\ Derivation of dimensionless numbers 3D kinematics}

The dimensionless numbers in Eqn 35 are derived by inserting the following expressions into Eqn 22 (as explained in the main text); $U \approx \sqrt{ }\left[U_{\infty}{ }^{2}+\left(4 \Phi_{0} R f\right)^{2}\right], \Omega=4 \Phi_{0} f$ and $\dot{\Omega}=2 \pi f \Omega$, which results in:

$$
\begin{aligned}
\frac{\dot{\Omega} R c}{U^{2}}= & \frac{2 \pi f \cdot 4 \Phi_{0} f \cdot R c}{U_{\infty}^{2}+\left(4 \Phi_{0} R f\right)^{2}} \cdot \frac{4 \Phi_{0} R}{4 \Phi_{0} R}= \\
& \frac{1}{\left(U_{\infty} / 4 \Phi_{0} R f\right)^{2}+1} \cdot \frac{2 \pi c}{4 \Phi_{0} R} \approx \frac{1}{J^{2}+1} \cdot \frac{c}{\Phi_{0} R}=\frac{1}{J^{2}+1} \cdot \frac{1}{A^{*}} .
\end{aligned}
$$

We took $\pi / 2 \approx 1$ instead of the more accurate $\pi / 2 \approx 1.5$, because it suffices for an order of magnitude analysis. Similarly, it follows that:

$$
\begin{aligned}
\frac{\Omega^{2} R c}{U^{2}}=\frac{\left(4 \Phi_{0} f\right)^{2} \cdot R c}{U_{\infty}^{2}+\left(4 \Phi_{0} R f\right)^{2}} \cdot \frac{R}{R}= & \\
& \frac{1}{\left(U_{\infty} / 4 \Phi_{0} R f\right)^{2}+1} \cdot \frac{c}{R}=\frac{1}{J^{2}+1} \cdot \frac{c}{R}=\frac{1}{J^{2}+1} \cdot \frac{1}{A R},
\end{aligned}
$$

$$
\begin{aligned}
\frac{\mu}{\rho U c}=\frac{v}{U c}= & \frac{v}{\sqrt{U_{\infty}^{2}+\left(4 \Phi_{0} R f\right)^{2} c}}= \\
& \frac{1}{\sqrt{\left(\frac{U_{\infty} c}{v}\right)^{2}+\left(\frac{4 \Phi_{0} R f}{v}\right)^{2}}}=\frac{1}{\sqrt{R e_{\mathrm{b}}^{2}+R e_{\mathrm{s}}^{2}}} .
\end{aligned}
$$

\section{D kinematics}

The dimensionless numbers is Eqn 47 are derived by inserting the following expressions into Eqn 22 to which $\mathbf{a}_{\text {wing }}$ is added (as explained in the main text); $U \approx \sqrt{ }\left[U_{\infty}{ }^{2}+(4 A f)^{2}\right], \dot{U}_{\text {wing }}=2 \pi f \cdot 4 A f$, $\Omega=4 \Phi_{0} f$ and $\dot{\Omega}=2 \pi f \Omega$, which results in:

$$
\begin{gathered}
\frac{\dot{U_{\text {wing }} c}}{U^{2}}=\frac{A 2 \pi 4 f^{2} c}{U^{2}}=\frac{A 2 \pi 4 f^{2} c}{U_{\infty}^{2}+(4 A f)^{2}}= \\
\frac{\frac{1}{J^{2}+1} \cdot \frac{A 2 \pi 4 c}{(4 A)^{2}} \approx \frac{1}{J^{2}+1} \cdot \frac{1}{A^{*}},}{\dot{\Omega} c^{2}} \frac{2 \pi f \cdot 4 \alpha_{0} f \cdot c^{2}}{U^{2}}=\frac{1}{U_{\infty}^{2}+(4 A f)^{2}}=\frac{2 \pi \cdot 4 \alpha_{0} \cdot c^{2}}{(4 A)^{2}} \approx \\
\frac{1}{J_{\infty}+1} \cdot \frac{\alpha_{0} \cdot c^{2}}{A^{2}}=\frac{1}{J^{2}+1} \cdot \frac{\alpha_{0}}{A^{* 2}} .
\end{gathered}
$$

Again, we took $\pi / 2 \approx 1$ instead of the more accurate $\pi / 2 \approx 1.5$, because it suffices for an order of magnitude analysis. Similarly, it follows that:

$$
\frac{\Omega^{2} c^{2}}{U^{2}}=\frac{\left(4 \alpha_{0} f\right)^{2} \cdot c^{2}}{U_{\infty}^{2}+(4 A f)^{2}}=\frac{1}{J^{2}+1} \cdot \frac{\alpha_{0}^{2} \cdot c^{2}}{(A)^{2}}=\frac{1}{J^{2}+1} \cdot \frac{\alpha_{0}^{2}}{A^{* 2}},
$$

$$
\begin{aligned}
& \frac{\mu}{\rho U c}=\frac{v}{U c}= \frac{v}{\sqrt{U_{\infty}^{2}+(4 A f)^{2} c}}= \\
& \frac{1}{\sqrt{\left(\frac{U_{\infty} c}{v}\right)^{2}+\left(\frac{4 A f c}{v}\right)^{2}}}=\frac{1}{\sqrt{R e_{\mathrm{b}}^{2}+R e_{\mathrm{s}}^{2}}} .
\end{aligned}
$$

\section{LIST OF ABBREVIATIONS}

$\alpha \quad$ wing angle of attack

$\dot{\alpha} \quad$ 1st time derivative of wing angle of attack

$\ddot{\alpha} \quad$ 2nd time derivative of wing angle of attack

$\alpha_{0} \quad$ wing angle of attack amplitude

$\alpha_{\text {eff }} \quad$ effective angle of attack

$\alpha_{\text {ind }} \quad$ induced angle of attack

$\alpha_{\text {geo }} \quad$ geometric angle of attack

$\beta \quad$ stroke plane angle

$\gamma \quad$ angle between the stroke plane and direction of flight

$\Delta r \quad$ width spanwise wing section

$\lambda \quad$ wingbeat wavelength

$\lambda * \quad$ dimensionless wavelength

$\lambda *_{0} \quad$ natural von Kármán vortex shedding wavelength

$\Lambda \quad$ dimensionless total stroke amplitude

dynamic viscosity

kinematic viscosity

flight path angle with respect to horizon

fluid density

wing deviation with respect to stroke plane 
wing stroke angle

1 st time derivative of wing stroke angle

2nd time derivative of wing stroke angle

total wing amplitude in radians

wing stroke amplitude (half the total stroke amplitude $\Phi$ )

absolute time-averaged angular velocity amplitude

absolute time-averaged angular acceleration amplitude

angular velocity of the rotating frame, and wing

angular acceleration of the rotating frame, and wing

amplitude of vector $\boldsymbol{\Omega}_{\text {wing }}$

angular velocity of the fly wing

dimensionless variable scaled with its order of magnitude

gradient (del) operator

angular acceleration

centripetal acceleration

Coriolis acceleration

acceleration with respect to inertial coordinate system

acceleration with respect to local coordinate system

linear acceleration of an airfoil

flap amplitude

stroke amplitude

single-wing aspect ratio

single-wing span

average wing (or foil) chord length

angular acceleration number

centripetal acceleration number

computational fluid dynamics

dimensionless circular distance moved during one full period (propeller)

total differential operator:

$$
\frac{D}{D t}=\frac{\partial}{\partial t}+\mathbf{u} \cdot \nabla
$$

Euler number

flap frequency

gravitational vector

advance ratio

reduced frequency

leading edge vortex

multiple (of $\lambda *_{0}$ )

Navier-Stokes

order of magnitude operator

pressure

ambient atmospheric pressure

magnitude of radius vector

position of a fluid particle in the rotating frame

wing radius

wing radius of gyration

Reynolds number

Reynolds number component due to body speed

Reynolds number component due to wings stroke,

Rossby number

dimensionless linear distance moved through air during one

full period (propeller)

number of chord lengths traveled at $R_{\mathrm{g}}$ during a full stroke

(Fig. 9)

magnitude of linear acceleration of the wing

single-wing area

outer surface of fly's body

outer boundary surface

Strouhal number

chord-based Strouhal number

time

velocity vector

velocity center of gravity of fly

velocity of fly at its outer surface

velocity with respect to inertial coordinate system $\mathbf{u}_{\text {loc }} \quad$ velocity in local coordinate system

$u_{\mathrm{n}} \quad$ component of wingtip speed normal to flight direction

$u_{\mathrm{t}} \quad$ component of wingtip speed in flight direction

$\mathbf{u}_{\alpha} \quad$ linear velocity distribution due to angle of attack variation

$\mathbf{u}_{\phi} \quad$ linear velocity distribution due to stroke

$\mathbf{u}_{\varphi} \quad$ linear velocity distribution due to deviation

$U \quad$ characteristic speed; absolute time-averaged speed of the wingtip

$U_{\text {body }} \quad$ amplitude of vector $\mathbf{u}_{\text {body }}$

$\dot{U}_{\text {wing }} \quad$ absolute time-averaged linear acceleration of the wing

$U_{\infty} \quad$ forward flight speed (arbitrary direction with respect to gravity)

V velocity along wing radius (Fig. 9)

$\mathbf{x} \quad$ position vector

$\ddot{x}_{\text {wing }} \quad x$-component of $\mathbf{a}_{\text {wing }}$

$(x, y, z) \quad$ local coordinate system

$(X, Y, Z) \quad$ inertial coordinate system

$\ddot{y}_{\text {wing }} \quad y$-component of $\mathbf{a}_{\text {wing }}$

We gratefully acknowledge Will Dickson, Koert Lindenburg and John Dabiri for proof reading preliminary versions of the manuscript. We thank Thomas Daniel for proof reading the final manuscript. We thank Johan van Leeuwen and GertJan van Heijst for hearty support, encouragement and proof reading of the various versions of the manuscript. This research is supported by NWO-ALW grant 817.02.012 to D.L. and by NSF grant IBN-0217229 to M.H.D.

\section{REFERENCES}

Altshuler, D. L., Dickson, W. B., Vance, J. T., Roberts, S. P. and Dickinson, M. H. (2005). Short-amplitude high-frequency wing strokes determine the aerodynamics of honeybee flight. Proc. Natl. Acad. Sci. USA 102, 18213-18218.

Anderson, J. D. (1991). Fundamentals of Aerodynamics. Boston, MA: McGraw-Hill.

Azuma, A. and Yasuda, K. (1989). Flight performance of rotary seeds. J. Theor. Biol. $138,23-53$.

Bandyopadhyay, P. R., Beal, D. N. and Menozzi, A. (2008). Biorobotic insights into how animals swim. J. Exp. Biol. 211, 206-214

Baruh, H. (1999). Analytical Dynamics. Boston, MA: McGraw-Hill.

Batchelor, G. K. (2000). Introduction to Fluid Dynamics. Cambridge: Cambridge University Press.

Birch, J. M., Dickson, W. B. and Dickinson, M. H. (2004). Force production and flow structure of the leading edge vortex on flapping wings at high and low Reynolds numbers. J. Exp. Biol. 207, 1063-1072.

Daniel, T. L. and Webb, P. W. (1987). Comparative Physiology: Life in Water and on Land (ed. P. Dejours, L. Bolis, C. R. Taylor and E. R. Weibel). Padova, Italy: Liviana Press.

Dickinson, M. H. (1994). The effects of wing rotation on unsteady aerodynamic performance at low Reynolds numbers. J. Exp. Biol. 192, 179-206.

Dickinson, M. H. and Goetz, K. G. (1993). Unsteady aerodynamic performance of model wings at low Reynolds numbers. J. Exp. Biol. 174, 45-64.

Dickinson, M. H., Lehmann, F. O. and Sane, S. P. (1999). Wing rotation and the aerodynamic basis of insect flight. Science 284, 1954-1960.

Dickson, W. B. and Dickinson, M. H. (2004). The effect of advance ratio on the aerodynamics of revolving wings. J. Exp. Biol. 207, 4269-4281.

Du, Z. and Selig, M. S. (1998). Effect of Rotation on the Boundary Layer of a Wind Turbine Blade. Bakersfield, CA: American Wind Energy Association, Windpower conference.

Dumitrescu, H. and Cardos, V. (2003). Rotational effects on the boundary-layer flow in wind turbines. AIAA J. 42, 408-411.

Ellington, C. P. (1984). The aerodynamics of insect flight I-VI. Philos. Trans. R. Soc. Lond. B Biol. Sci. 305, 1-181.

Ellington, C. P., Van den Berg, C., Willmott, A. P. and Thomas, A. L. R. (1996). Leading-edge vortices in insect flight. Nature 384, 626-630.

Fry, S. N., Sayaman, R. and Dickinson, M. H. (2003). The aerodynamics of free-flight maneuvers in Drosophila. Science 300, 495-498.

Green, S. I. (1995). Vortex Dynamics in the Wake of a Cylinder. Dordrecht: Kluwer Academic Publishers.

Greitzer, E. M., Tan, C. S. and Graf, M. B. (2004). Internal Flow Concepts and Applications. Cambridge: Cambridge University Press.

Guyon, E., Hulin, J. P., Petit, L. and Mitescu, C. D. (2001). Physical Hydrodynamics. Oxford: Oxford University Press.

Himmelskamp. H. (1947). Profile investigations on a rotating airscrew. Dissertation Göttingen 1945, Reports and Translations No. 832.

Hubel, T. (2006). Untersuchungen zur instationären Aerodynamik an einem vogelähnlichen Flügelschlagmodell, $\mathrm{PhD}$ thesis, TU Darmstadt, Fachbereich Biologie.

Lentink, D. (2003). Influence of airfoil shape on performance in insect flight. MSc.Thesis, Delft University of Technology (fac. AE).

Lentink, D. and Dickinson, M. H. (2009). Rotational accelerations stabilize leading edge vortices on revolving fly wings. J. Exp. Biol. 212, 2705-2719.

Lentink, D. and Gerritsma, M. I. (2003). Influence of airfoil shape on performance in insect flight. AIAA 2003-3447.

Lentink, D., Muijres, F. T., Donker-Duyvis, F. J. and van Leeuwen, J. L. (2008). Vortex-wake interactions of a flapping foil that models animal swimming and flight. J. Exp. Biol. 211, 267-273. 
Lindenburg, C. (2004). Modelling of rotational augmentation based on engineering considerations and measurements. European Wind Energy Conference, London.

Liu, H. and Kawachi, K. (1998). A numerical study of insect flight. J. Comput. Phys. 146, 124-156.

Maxworthy, T. (1979). Experiments on the Weis-Fogh mechanism of lift generation by insects in hovering flight. Part 1. Dynamics of the 'fling'. J. Fluid Mech. 93, 47-63.

Ponta, F. L. and Aref, H. (2005). Vortex synchronization regions in shedding from an oscillating cylinder. Phys. Fluids 17, 011703.

Reynolds, O. (1883). An experimental investigation of the circumstances which determine whether the motion of water shall be direct or sinuous, and of the law of resistance in parallel channels. Philos. Trans. R. Soc. Lond. B Biol. Sci. 174, 935-982.

Rossby, C. G. (1936). On the momentum transfer at the sea surface. Part I Papers Phys Oceanogr Meteorol 3 no 3

Sane, S. P. and Dickinson, M. H. (2001). The control of flight force by a flapping wing: lift and drag production. J. Exp. Biol. 204, 2607-2626.

Schlichting, H. (1979). Boundary-Layer Theory, 7th edn. New York: McGraw-Hill.

Sun, M. and Tang, J. (2002). Lift and power requirements of hovering flight in Drosophila virilis. J. Exp. Biol. 205, 2413-2427.

Tangler, J. L. (2004). Insight into wind turbine stall and post-stall aerodynamics. Wind Energy 7, 247-260.

Taylor, G. K., Nudds, R. L. and Thomas, A. L. R. (2003). Flying and swimming animals cruise at a Strouhal number tuned for high power efficiency. Nature $\mathbf{4 2 5}$, 707-711.
Tennekes, H. and Lumley, J. L. (1983). A First Course in Turbulence. Cambridge, MA: MIT Press.

Tobalske, B. W., Warrick, D. R., Clark, C. J., Powers, D. R., Hedrick, T. L., Hyder, G. A. and Biewener, A. A. (2007). Three-dimensional kinematics of hummingbird flight. J. Exp. Biol. 210, 2368-2382.

Triantafyllou, G. S., Triantafyllou, M. S. and Grosenbaugh, M. A. (1993). Optima

thrust development in oscillating foils with application to fish propulsion. J. Fluid Struct. 7, 205-224.

Tritton, D. J. (2005). Physical Fluid Dynamics. Oxford: Oxford University Press.

Usherwood, J. R. and Ellington, C. P. (2002). The aerodynamics of revolving wings I-II. J. Exp. Biol. 205, 1547-1576.

Vanyo, J. P. (1993). Rotating Fluids in Engineering and Science. Mineola, NY: Dover Press.

Vogel, S. (1994). Life in Moving Fluids. Princeton, NJ: Princeton University Press

Wang, Z. J. (2000a). Two dimensional mechanism for insect hovering. Phys. Rev. Lett. 85, 2216-2218.

Wang, Z. J. (2000b). Shedding and frequency selection in flapping flight. J. Fluid. Mech. 410, 323-341.

Weis-Fogh, T. (1973). Quick estimates of flight fitness in hovering animals, including novel mechanisms for lift production. J. Exp. Biol. 59, 169-230.

White, F. M. (1991). Viscous Fluid Flow. New York: McGraw-Hill.

Williamson, C. H. K. and Roshko, A. (1988). Vortex formation in the wake of an oscillating cylinder. J. Fluid Struct. 2, 355-381. 